\title{
Collective modes in the fluxonium qubit
}

\author{
Giovanni Viola \\ Institute for Quantum Information, RWTH Aachen University, 52056 Aachen, Germany \\ and Department of Microtechnology and Nanoscience (MC2), Chalmers University of Technology, SE-412 96 Gothenburg, Sweden \\ Gianluigi Catelani* \\ Peter Grünberg Institut (PGI-2) and JARA Institute for Quantum Information, Forschungszentrum Jülich, 52425 Jülich, Germany
}

(Received 29 June 2015; revised manuscript received 30 October 2015; published 21 December 2015)

\begin{abstract}
Superconducting qubit designs vary in complexity from single- and few-junction systems, such as the transmon and flux qubits, to the many-junction fluxonium. Here, we consider the question of whether the many degrees of freedom in the fluxonium circuit can limit the qubit coherence time. Such a limitation is in principle possible, due to the interactions between the low-energy, highly anharmonic qubit mode and the higher-energy, weakly anharmonic collective modes. We show that so long as the coupling of the collective modes with the external electromagnetic environment is sufficiently weaker than the qubit-environment coupling, the qubit dephasing induced by the collective modes does not significantly contribute to decoherence. Therefore, the increased complexity of the fluxonium qubit does not constitute by itself a major obstacle for its use in quantum computation architectures.
\end{abstract}

DOI: 10.1103/PhysRevB.92.224511

\section{INTRODUCTION}

Starting from the pioneering experiments with Cooper pair boxes [1], superconducting qubits have been vastly improved [2], thanks in part to better control of the qubit environment. For example, three-dimensional (3D) transmons [3] benefit from being placed in a cavity that suppresses radiative losses as well as from their relatively large size that decreases the role of surface dielectric losses. Planar transmon variants such as the Xmon [4] have shorter coherence times, but have made possible demonstrations of the building blocks of quantum error correction codes [5-8]. In contrast to the transmon architecture, in which a Josephson junction is shunted capacitively, in a fluxonium qubit the shunt is via an inductance $[9,10]$. To realize in practice a sufficiently large inductance, arrays with many Josephson junctions are used. Even in this more complicated circuit, long relaxation times have been achieved, so long, in fact, to make possible the accurate measurement of the phase dependence of the quasiparticle dissipation through a Josephson junction [11]. Experimentally, the fluxonium coherence time is much shorter than the limit imposed by relaxation; in this paper, we theoretically investigate whether the complexity of the circuit, arising from the use of junction arrays, contributes to this shortness. Along the way, we derive approximate Hamiltonians for a few variants of the fluxonium circuit, demonstrating the validity of the approximations for current experimental setups.

The physics of arrays made of identical Josephson junctions has long attracted the interest of theorists and experimentalists alike, especially in the context of the superconductor-insulator transition controlled by the ratio of charging and Josephson energies $[12,13]$. More recently, renewed attention has been given to the physics of collective modes, whose frequency can be significantly lowered below the constituent Josephson junctions plasma frequency due to ground capacitances; in particular, the interplay between collective modes and quantum

\footnotetext{
*g.catelani@fz-juelich.de
}

PACS number(s): 74.50.+r, 85.25.Cp

phase slips in rings has been studied [14-16], as well as the localizing effect of disorder in the junction parameters [17]. Long junction arrays can have a large inductance, proportional to the number of elements, and for quantum computation applications such superinductors [18] have been proposed as elements of topologically protected qubits [19], for example, the $0-\pi$ qubit $[20,21]$. An alternative design for a superinductor has also been realized [22] and recently implemented in a qubit circuit [23]. In the fluxonium, even in the ideal case of no capacitances to ground, the full symmetry of a ring is broken by the presence of a smaller junction. Nonetheless, in the ideal case a permutation symmetry is still present; the effect of this symmetry and its breaking on the fluxonium spectrum has been studied in Ref. [24]. Aspects of the quasiparticle-induced decoherence in the fluxonium have been studied theoretically in Refs. [25-27].

In this paper, we focus on the possible limitation of the coherence of the qubit mode due to its interactions with the collective modes of the array; we will show that both ground capacitances and array-junction nonlinearities introduce potential decoherence channels. While they are not currently limiting the fluxonium coherence, we nonetheless point out that isolation of the collective modes from the electromagnetic environment is necessary for long coherence times. Interestingly, simple variations in the design of the circuit (such as the placement of capacitors used to couple to the qubit) can significantly affect the decoherence rate. In the next section, we discuss the mechanism by which the collective modes can lead to dephasing of the qubit and which quantities determine the dephasing rate. Throughout the paper, we set $\hbar=1$.

\section{COLLECTIVE MODES AND QUBIT DEPHASING}

To understand how the qubit-collective modes interactions can affect coherence, let us briefly review the so-called photon shot noise (or measurement-induced) dephasing, originating from the interaction between a qubit and the electromagnetic modes of the cavity in which it is placed [28,29]. As a 
consequence of this coupling, the qubit frequency $\omega_{10}$ depends on the occupation of the cavity modes

$$
\omega_{10}\left(\left\{n_{\mu}\right\}\right)=\omega_{10}+2 \sum_{\mu} \bar{\chi}_{\mu} n_{\mu},
$$

where $n_{\mu}$ is the occupation number of mode $\mu$ and $\bar{\chi}_{\mu}$ denotes the dispersive shift of that mode.

The fluctuations of the occupations of the modes cause fluctuations in the qubit frequency and hence dephasing. The photon shot-noise dephasing has been investigated in detail in the 3D transmon architecture [30,31]. In particular, in Ref. [30] good agreement between theory and experiment was found over a range of average occupation number in the cavity $\bar{n}$ from small to relatively large $(\bar{n} \sim 3)$, and a residual occupation of order $1 \%$ was estimated. As we will show in the following sections, in the fluxonium the collective modes of the junction array are coupled to the qubit in a way that leads to dispersive shifts of the qubit frequency. The collective modes are also (weakly) coupled to the cavity (see Sec. IV C) and therefore their residual occupation is small. Hence, we restrict ourselves to the relevant case of small occupation number $\bar{n}_{\mu} \ll 1$ for any mode. In this case, each collective mode contributes a rate [31]

$$
\Gamma_{\mu}=\frac{4 \kappa_{\mu} \bar{\chi}_{\mu}^{2}}{\kappa_{\mu}^{2}+4 \bar{\chi}_{\mu}^{2}} \bar{n}_{\mu},
$$

with $\kappa_{\mu}$ the decay rate of mode $\mu$ [32], to the total qubit dephasing rate

$$
\Gamma_{\phi}=\sum_{\mu} \Gamma_{\mu} .
$$

Equation (2) was derived assuming that the effect of each mode can be treated independently [31]. If the dispersive shifts are known, this expression enables us to put an upper limit on the dephasing rate since independently of $\kappa_{\mu}$, the rate satisfies $\Gamma_{\mu} \leqslant\left|\bar{\chi}_{\mu}\right| \bar{n}_{\mu}$. The inequality is saturated for $\kappa_{\mu}=2\left|\bar{\chi}_{\mu}\right|$, while in the limiting cases of $\kappa_{\mu}$ much bigger or smaller than $\left|\bar{\chi}_{\mu}\right|$ we have the approximate relations

$$
\Gamma_{\mu} \simeq \begin{cases}\frac{4 \bar{\chi}_{\mu}^{2}}{\kappa_{\mu}} \bar{n}_{\mu}, \quad \kappa_{\mu} \gg\left|\bar{\chi}_{\mu}\right| \\ \kappa_{\mu} \bar{n}_{\mu}, \quad \kappa_{\mu} \ll\left|\bar{\chi}_{\mu}\right| .\end{cases}
$$

We see that to calculate the dephasing rate $\Gamma_{\phi}$ we need to know both $\bar{\chi}_{\mu}$ and $\kappa_{\mu}$. Our goal is to estimate these quantities for the collective modes of the fluxonium.

In the remainder of the paper we study a realistic model of the qubit (see Sec. III); it includes ground and coupling capacitors in addition to the charging and Josephson energies of each junction. The interactions between collective and qubit modes due to ground capacitances is the subject of Sec. IV, where we also discuss the collective modes decay rates. In Sec. V, we consider the effects of the array-junction nonlinearity. Some consequences of breaking the circuit parity symmetry and placing the coupling capacitors in the array are briefly discussed in Secs. VI and VII, respectively. Finally, in Sec. VIII using the results of the previous sections we estimate the fluxonium dephasing rate due to the interactions with the collective modes. We summarize our main results in Sec. IX. Numerous technical details are presented in Appendixes A through $\mathrm{H}$.

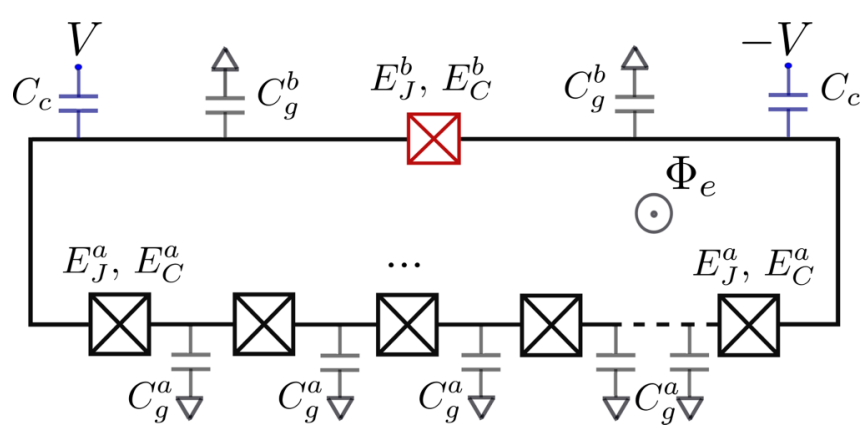

FIG. 1. (Color online) Circuit model for the fluxonium: a small junction (top, Josephson energy $E_{J}^{b}$, charging energy $E_{C}^{b}$ ) shunts an array (bottom) of many identical junctions. A capacitance $\left(C_{g}^{a}\right.$ or $C_{g}^{b}$ ) is present between each superconducting island and ground. Additional capacitors $C_{c}$, biased at voltages $\pm V$, are used to control and read out the qubit, whose spectrum depends on the applied flux $\Phi_{e}$.

\section{FLUXONIUM MODEL}

The circuit model for the fluxonium qubit is shown in Fig. 1: the two ends of an array of $N \gg 1$ identical Josephson junctions (Josephson energy $E_{J}^{a}$ and charging energy $E_{C}^{a}=$ $\left.e^{2} / 2 C_{J}^{a}\right)$ are connected by a so-called phase-slip junction (Josephson energy $E_{J}^{b}$ and charging energy $E_{C}^{b}$ ). The loop thus formed is pierced by a magnetic flux $\Phi_{e}$. To suppress phase fluctuations in the array, we require $E_{J}^{a} / E_{C}^{a} \gg 1$; phase slips preferentially take place at the phase-slip junction so long as $E_{J}^{b} / E_{C}^{b}<E_{J}^{a} / E_{C}^{a}$. The superconducting islands inside the array have ground capacitances $C_{g}^{a}$ and those at the ends $C_{g}^{b}$. Identical coupling capacitors of capacitance $C_{c}$, used to control the system by applying ac voltages $+V$ and $-V$ to them, are connected to the islands at the end of the array. The Lagrangian description of this circuit without the coupling capacitors is discussed in detail in Ref. [24]. In Appendix A, we briefly summarize (for the paper to be self-contained) and generalize (to include the coupling capacitors) the relevant parts of that work.

While there are $N+1$ junctions in the circuit, due to charge conservation and flux quantization there are only $N$-independent degrees of freedom [24]: the qubit mode $\phi$ and the collective modes $\xi_{\mu}, \mu=1, \ldots, N-1$. In terms of these modes, the Lagrangian $\mathcal{L}_{S}$ in the absence of ground and coupling capacitors takes the form

$$
\begin{gathered}
\mathcal{L}_{S}=T_{S}-U_{S}, \\
T_{S}=\frac{1}{16 E_{C}^{\phi}} \dot{\phi}^{2}+\frac{1}{16 E_{C}^{a}} \sum_{\mu} \dot{\xi}_{\mu}^{2}, \\
U_{S}=-E_{J}^{b} \cos \left(\phi+\varphi_{e}\right) \\
-E_{J}^{a} \sum_{m} \cos \left[\frac{\phi}{N}+\sum_{\mu} W_{\mu m} \xi_{\mu}\right],
\end{gathered}
$$

where the phase bias $\varphi_{e}=2 \pi \Phi_{e} / \Phi_{0}$ is due to the externally applied magnetic flux $\Phi_{e}$, with $\Phi_{0}$ the flux quantum, and the qubit mode charging energy $E_{C}^{\phi}$ is

$$
\frac{1}{E_{C}^{\phi}}=\frac{1}{E_{C}^{b}}+\frac{1}{N E_{C}^{a}} ;
$$


note that due to the last term in the above equation, $E_{C}^{\phi}$ is smaller than the phase-slip junction charging energy $E_{C}^{b}$. Hereinafter, sums over index $m$ run from 1 to $N$ and those over Greek indices such as $\mu$ from 1 to $N-1$. To write the Lagrangian in the given form, the matrix $W_{\mu m}$ must satisfy $\sum_{m} W_{\mu m} W_{\nu m}=\delta_{\mu \nu}$ and $\sum_{m} W_{\mu m}=0$; for concrete calculations, we will use for $W_{\mu m}$ the form suggested in Ref. [24]:

$$
W_{\mu m}=\sqrt{\frac{2}{N}} \cos \frac{\pi \mu(m-1 / 2)}{N} .
$$

It can be shown [24] that $\mathcal{L}_{S}$ is symmetric under the action of the symmetric group $\mathrm{S}_{N}$.

Due to the large ratio $E_{J}^{a} / E_{C}^{a}$, fluctuations of $\xi_{\mu}$ are small; for fluctuations in $\phi$ small compared to $\pi N$, we can then expand the last term in $U_{S}$ to quadratic order to find (up to a constant term)

$$
U_{S} \simeq U=-E_{J}^{b} \cos \left(\phi+\varphi_{e}\right)+\frac{1}{2} E_{L} \phi^{2}+\frac{1}{2} E_{J}^{a} \sum_{\mu} \xi_{\mu}^{2}
$$

with $E_{L}=E_{J}^{a} / N$. At this lowest order, the qubit mode $\phi$ and collective modes $\xi_{\mu}$ do not interact (so long as we neglect ground and coupling capacitors), and the Lagrangian

$$
\mathcal{L}_{U}=T_{S}-U
$$

is symmetric under the unitary group $\mathrm{U}(N-1)$. We note that the symmetry under permutations ensures [24] that the anharmonic terms that we neglect cannot couple any state given by the direct product between a qubit eigenstate and a collective modes singly excited state to a state which is the direct product between any qubit state and the collective modes ground state. One of the main objectives of this work is to understand the interactions induced by the presence of ground and coupling capacitors; we will show, for example, that the coupling between the states just discussed is in general present, albeit weak.

Ground and coupling capacitors modify the kinetic energy part of the Lagrangian by the addition of the term $T_{G}$ given by

$$
T_{G}=\frac{1}{16}\left[G_{00} \dot{\phi}^{2}+2 \sum_{\mu} G_{0 \mu} \dot{\phi} \dot{\xi}_{\mu}+\sum_{\mu \nu} G_{\mu \nu} \dot{\xi}_{\mu} \dot{\xi}_{\nu}\right],
$$

where the symmetric matrix $G$ has entries

$$
\begin{gathered}
G_{00}=\frac{1}{4 E_{t}}\left[1-\frac{2}{3} \frac{N+1}{N} \lambda\right], \\
G_{0 \mu}=-\frac{1}{2 E_{g}^{a}} \frac{c_{\mu} o_{\mu+1}}{\sqrt{2 N} s_{\mu}^{2}}, \\
G_{\mu \nu}=\frac{1}{4 E_{g}^{a}} \frac{1}{s_{\mu}^{2}}\left[\delta_{\mu \nu}-\lambda \frac{2}{N(N-1)} \frac{o_{\mu} o_{\nu} c_{\mu} c_{\nu}}{s_{\nu}^{2}}\right] .
\end{gathered}
$$

Here, the energy scales are $E_{g}^{a}=e^{2} / 2 C_{g}^{a}$ and $E_{t}=e^{2} / 2 C_{t}$, where the total capacitance $C_{t}$ is

$$
C_{t}=2\left(C_{g}^{b}+C_{c}\right)+(N-1) C_{g}^{a} .
$$

The dimensionless parameter $0 \leqslant \lambda \leqslant 1$ is defined as

$$
\lambda=\frac{(N-1) C_{g}^{a}}{C_{t}}
$$

TABLE I. Fluxonium parameters used for numerical calculations throughout the paper (see also Appendix B). Energies are given in $\mathrm{GHz}$, while the number of junctions $N, \lambda$ [Eq. (17)] and the screening length $N_{s}$ [Eq. (27)] are dimensionless.

\begin{tabular}{ccccccccccc}
\hline \hline & $N$ & $E_{J}^{a}$ & $E_{C}^{a}$ & $E_{J}^{b}$ & $E_{C}^{b}$ & $E_{g}^{a}$ & $E_{g}^{b}$ & $E_{C}^{c}$ & $\lambda$ & $N_{s}$ \\
\hline Set 1 & 43 & 26.0 & 1.24 & 8.93 & 3.60 & 194 & 6 & 24.2 & 0.34 & 39 \\
Set 2 & 95 & 48.3 & 1.01 & 10.2 & 4.78 & 484 & 807 & 12.1 & 0.54 & 69 \\
\hline \hline
\end{tabular}

and we introduced the shorthand notations

$$
\begin{gathered}
s_{\mu}=\sin \frac{\pi \mu}{2 N}, \\
c_{\mu}=\cos \frac{\pi \mu}{2 N}, \\
o_{\mu}=\frac{1-(-1)^{\mu}}{2} .
\end{gathered}
$$

The coupling capacitors enable control of the circuit via external ac voltages; the coupling Lagrangian $\mathcal{L}_{V}$ takes the form

$$
\mathcal{L}_{V}=-\frac{1}{4 E_{C}^{c}} \dot{\phi} e V,
$$

where we assumed that the voltages applied to the capacitors are equal in magnitude $V$ and opposite in sign and $E_{C}^{c}=$ $e^{2} / 2 C_{c}$

From the above definitions, it is evident that the collective modes with even index $\mu$ interact only with the qubit mode, whereas the odd modes interact only among themselves. Therefore, the total approximate [33] Lagrangian $\mathcal{L}$ separates into a sum of even and odd sectors:

$$
\mathcal{L}=\mathcal{L}_{U}+T_{G}+\mathcal{L}_{V}=\mathcal{L}_{e}+\mathcal{L}_{o} .
$$

This separation is valid as long as parity $P$ and time-reversal $T$ symmetries are preserved [24]: indeed, the qubit mode and the collective modes with even index $\mu$ are even under $P T$ symmetry, while modes with odd index are odd. We restrict our attention to the $P T$-symmetric case for most of the paper, but we discuss the consequences of breaking this symmetry in Sec. VI. In the next section, we focus on the even sector. The analysis of the odd-sector modes, which are decoupled from the qubit, is in Appendix C. Throughout the paper, we will present examples calculated with the two-parameter set given in Table I; as explained in Appendix B, the parameters are chosen as to reflect realistic experimental values $[9,11]$.

\section{EVEN SECTOR AND QUBIT MODE}

The qubit mode $\phi$ belongs to the even sector, and in the presence of capacitance to ground in the array it interacts with all the even modes:

$$
\begin{aligned}
\mathcal{L}_{e}= & \mathcal{L}_{\phi}+T_{e}-\frac{1}{2} E_{J}^{a} \sum_{\rho=1}^{N_{e}} \eta_{\rho}^{2}+\mathcal{L}_{V} \\
& -\frac{1}{16 \sqrt{2 N}} \frac{1}{E_{g}^{a}} \dot{\phi} \sum_{\rho=1}^{N_{e}} \frac{c_{2 \rho}}{s_{2 \rho}^{2}} \dot{\eta}_{\rho} .
\end{aligned}
$$


Here, we define $\eta_{\rho}=\xi_{2 \rho}$ and the number of even modes is $N_{e}=\lfloor(N-1) / 2\rfloor$ (with $\lfloor x\rfloor$ the integer part of $x$ ). The kinetic energy of the even modes has a simple diagonal form

$$
T_{e}=\frac{1}{16} \sum_{\rho=1}^{N_{e}}\left[\frac{1}{E_{C}^{a}}+\frac{1}{4 E_{g}^{a}} \frac{1}{s_{2 \rho}^{2}}\right] \dot{\eta}_{\rho}^{2} \equiv \frac{1}{16} \sum_{\rho=1}^{N_{e}} \frac{1}{E_{C, \rho}^{e}} \dot{\eta}_{\rho}^{2}
$$

and the qubit Lagrangian is

$$
\mathcal{L}_{\phi}=\frac{1}{16 \tilde{E}_{C}^{\phi}} \dot{\phi}^{2}+E_{J}^{b} \cos \left(\phi+\varphi_{e}\right)-\frac{1}{2} E_{L} \phi^{2},
$$

where the effective qubit charging energy is given by

$$
\frac{1}{\tilde{E}_{C}^{\phi}}=\frac{1}{E_{C}^{\phi}}+\frac{1}{4 E_{t}}\left(1-\frac{2}{3} \frac{N+1}{N} \lambda\right) .
$$

The Lagrangian $\mathcal{L}_{e}$ has a simple structure, describing a set of independent harmonic oscillators interacting with the qubit mode. To this Lagrangian, however, corresponds an Hamiltonian $H_{e}$ in which all degrees of freedom interact among themselves (see Appendix D) due the nondiagonal form of the kinetic energy [cf. the last term in Eq. (23)]. If the number of junctions is not too large,

$$
N \ll N_{s} \equiv \pi\left(\frac{E_{g}^{a}}{E_{C}^{a}}\right)^{1 / 2},
$$

the effect of the ground capacitances can be treated perturbatively for all modes; indeed, if the array is short compared to the "screening length" $N_{s}$, the ground capacitances hardly affect the energy spectrum of the modes [14]. However, the condition (27) is not satisfied in current experiments (see Table I). As detailed in Appendix D, we find that the generally weaker condition

$$
N \ll 8 \pi^{2} \frac{E_{g}^{a}}{\tilde{E}_{C}^{\phi}}
$$

enables us to make substantial simplifications. This condition is satisfied in current experiments, with the right-hand side being about 6400 (10600) for parameter set 1 (2). The approximate Hamiltonian then takes the form

$$
\begin{gathered}
H_{e}=H_{\phi}+\sum_{\rho=1}^{N_{e}} H_{\rho}+H_{\mathrm{int}}+H_{V}, \\
H_{\phi}=4 \tilde{E}_{C}^{\phi} p_{\phi}^{2}-E_{J}^{b} \cos \left(\phi+\varphi_{e}\right)+\frac{1}{2} E_{L} \phi^{2}, \\
H_{\rho}=4 E_{C, \rho}^{e} p_{\rho}^{2}+\frac{1}{2} E_{J}^{a} \eta_{\rho}^{2}, \\
H_{\mathrm{int}}=\sum_{\rho=1}^{N_{e}} g_{\rho} p_{\rho} p_{\phi}, \quad g_{\rho}=\frac{4}{\sqrt{2 N}} \frac{\tilde{E}_{C}^{\phi} E_{C, \rho}^{e}}{E_{g}^{a}} \frac{c_{2 \rho}}{s_{2 \rho}^{2}}, \\
H_{V}=-2 \frac{\tilde{E}_{C}^{\phi}}{E_{C}^{c}} p_{\phi} e V-\sum_{\rho=1}^{N_{e}} \frac{1}{4 E_{C}^{c}} g_{\rho} p_{\rho} e V .
\end{gathered}
$$

Here, $p_{\rho}$ is the momentum conjugate to $\eta_{\rho}$. The above expression for the Hamiltonian is one of the main results of this paper: it contains the leading interaction terms between the qubit mode and the collective modes of the junctions forming the superinductance due to capacitance to ground

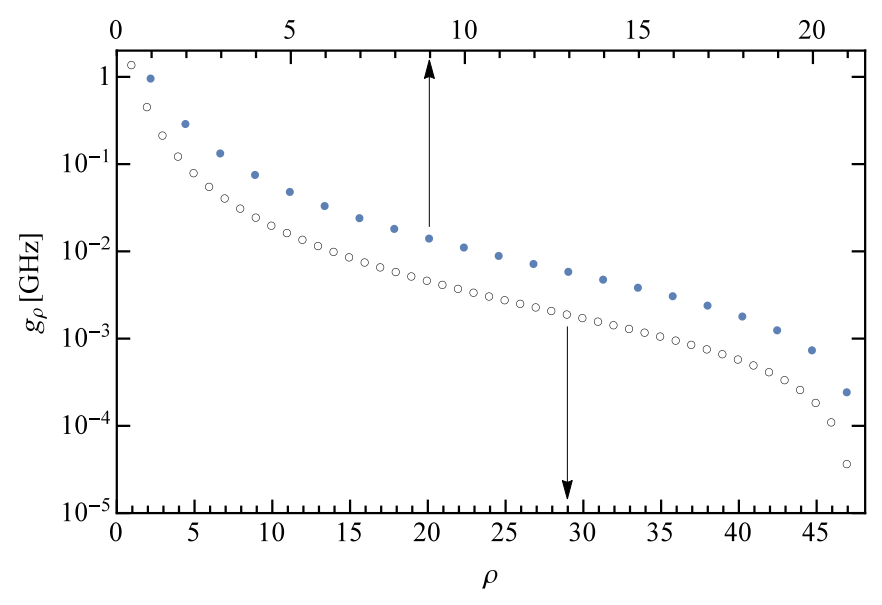

FIG. 2. (Color online) Qubit-collective modes coupling constants $g_{\rho}$ [Eq. (32)], calculated using parameter sets 1 in Table I (filled circles) and 2 (empty circles). Different horizontal scales are used for the two sets, as indicated by the arrows, and hence $g_{\rho}$ for set 2 is larger than the corresponding coupling constant for set 1 . Note that in absolute value the coupling strength between qubit and the few lowest even modes is as strong as or even stronger than the typical qubit-cavity coupling $[9,10]$.

in the array. (Interactions arising from the array junction nonlinearity are discussed in Sec. V.) The coupling constant $g_{\rho}$ is proportional to the array capacitance to ground and monotonically decreases with $\rho$ (see Fig. 2); therefore, the higher collective modes couple more weakly to the qubit. Similarly, since $g_{\rho}$ determines also the coupling of the collective modes to the ac voltage $V$, the higher modes are more weakly coupled to $V$ than the lower ones; moreover, the low-energy ones are more weakly coupled to $V$ than the qubit mode. As we will see in the following, this implies a lower decay rate for the collective modes compared to the qubit.

We note that the capacitances to ground $C_{g}^{b}$ of the islands next to the phase-slip junction (see Fig. 1) do not enter into the interaction Hamiltonian $H_{\text {int }}$, while they affect the effective qubit charging energy $\tilde{E}_{C}^{\phi}[$ Eq. (26)] via the parameter $\lambda$ of Eq. (17). Therefore, interaction strength and qubit frequency can in principle be tuned independently by changing the ratio $C_{g}^{b} / C_{g}^{a}$

\section{A. Dispersive shifts}

To further study the effect on the qubit of the interaction with the collective modes, we perform a Schrieffer-Wolff transformation and project the Hamiltonian $H_{e}$ [Eq. (29)] onto the qubit subspace to find the effective Hamiltonian [34]

$$
H_{\mathrm{eff}}=\frac{\omega_{10}(f)}{2} \sigma^{z}+\sum_{\rho=1}^{N_{e}}\left[\omega_{\rho}+\chi_{\rho}(f) \sigma^{z}\right] a_{\rho}^{\dagger} a_{\rho},
$$

where $\sigma^{z}$ is a Pauli matrix in the qubit subspace,

$$
\omega_{\rho}^{e}=\sqrt{8 E_{C, \rho}^{e} E_{J}^{a}}
$$

is the harmonic oscillator frequency of the even collective mode $\rho$, and $a_{\rho}^{\dagger}, a_{\rho}$ the creation and annihilation operators. As indicated by the presence of the parameter $f=\varphi_{e} / 2 \pi$, the 


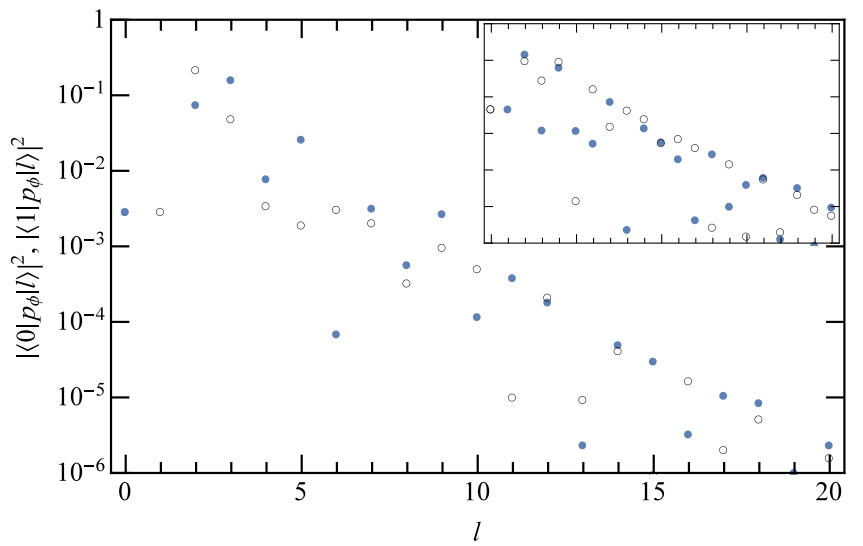

FIG. 3. (Color online) Main panel: matrix elements squared $\left|\left\langle 0\left|p_{\phi}\right| l\right\rangle\right|^{2}$ (filled circles) and $\left|\left\langle 1\left|p_{\phi}\right| l\right\rangle\right|^{2}$ (empty circles) at $f=0.35$ for $l \leqslant 20$ calculated using parameter set 1 (see Table I). Inset: same as main panel but for parameter set 2 . We stress that the overall decrease of the matrix elements with $l$ is valid for any flux $f$, not just for the particular value used here as an example.

qubit frequency $\omega_{10}$ and the ac Stark shifts $\chi_{\rho}$ depend on flux through the loop, and we neglect for the moment the coupling to external bias given by $H_{V}$. Here, with the frequency $\omega_{10}$ we indicate the energy difference between the two lowest eigenstates of the Hamiltonian $H_{\phi}$ [Eq. (30)]; following, we will discuss a small renormalization $\delta \omega_{10}$ of the qubit frequency due to the interaction with the collective modes.

The dispersive shifts $\chi_{\rho}$ depend on matrix elements of charge operator $p_{\phi}$ that involve all the eigenstates $|l\rangle$ with energy $\epsilon_{l}(l=0,1,2, \ldots)$ of Hamiltonian $H_{\phi}[34]$ :

$$
\begin{aligned}
\chi_{\rho}= & \frac{1}{2} \sqrt{\frac{E_{J}^{a}}{8 E_{C, \rho}^{e}}} g_{\rho}^{2}\left[\left|\left\langle 0\left|p_{\phi}\right| 1\right\rangle\right|^{2} \frac{2 \omega_{10}}{\omega_{10}^{2}-\omega_{\rho}^{2}}\right. \\
& +\sum_{l \geqslant 2}\left|\left\langle 0\left|p_{\phi}\right| l\right\rangle\right|^{2} \frac{\omega_{l 0}}{\omega_{l 0}^{2}-\omega_{\rho}^{2}} \\
& \left.-\sum_{l \geqslant 2}\left|\left\langle 1\left|p_{\phi}\right| l\right\rangle\right|^{2} \frac{\omega_{l 1}}{\omega_{l 1}^{2}-\omega_{\rho}^{2}}\right]
\end{aligned}
$$

with $\omega_{l j}=\epsilon_{l}-\epsilon_{j}$. While in the case of the transmon [35] selection rules and low anharmonicity enable the analytical calculation of the dispersive shift, for the fluxonium this is not possible, so we resort to numerical estimates. Fast convergence of the sums in the above equation is ensured by the rapid decrease of the matrix elements $\left\langle 0\left|p_{\phi}\right| l\right\rangle$ and $\left\langle 1\left|p_{\phi}\right| l\right\rangle$ as $l$ increases (see Fig. 3); further aiding the convergence is the approximately linear increase of the energy of the states with slope $\sim\left(8 \tilde{E}_{C}^{\phi} E_{L}\right)^{1 / 2}$. The reason for the decay of the matrix elements is the following: the low-lying states are localized near $\phi_{e}$, while the high-energy states are to a good approximation the eigenstates of the harmonic oscillator obtained by neglecting the Josephson term in $H_{\phi}$ (this also explains the linear increase in their energy). Since these high-energy states display oscillations of small magnitude at the center of the potential, the overlap of their derivative with the low-lying states is small, and the increase of the number of oscillations with $l$ causes the decrease of the matrix elements.

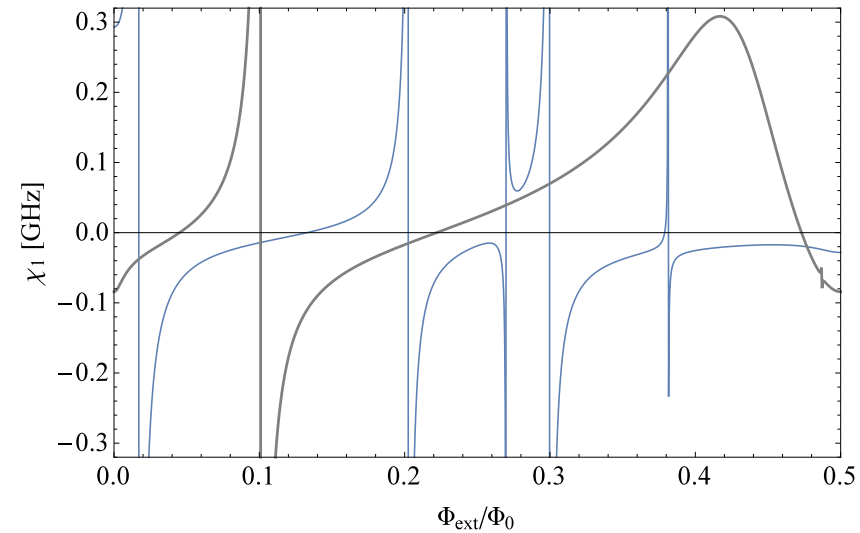

FIG. 4. (Color online) Dispersive shift $\chi_{1}$ [Eq. (36)] as function of external flux for parameter sets 1 (thin line) and 2 (thick line). Note that the vertical scale covers a range (in absolute value) approximately a factor of 3 smaller than the coupling constant $g_{1}$ (cf. Fig. 2). Since higher-order corrections neglected here are suppressed by a factor of order $\left(\chi_{1} / g_{1}\right)^{2}$ [34], near the limits of this range quantitative corrections to the calculated values are possible, in particular near the resonances.

The dispersive shifts calculated via Eq. (36) diverge as the energy differences $\omega_{l 0}$ or $\omega_{l 1}$ approach one of the collective modes frequencies $\omega_{\rho}$. This divergence, however, only signals the breakdown of the perturbative calculation near such resonant conditions: the actual dispersive shift is limited in magnitude by the coupling constant $g_{\rho}$, so validity of the perturbative approach is given by the condition $\left|\chi_{\rho}\right| \ll g_{\rho}$ [36]. Despite this limitation, Eq. (36) correctly estimates the dispersive shifts at most flux values. We show in Figs. 4 and 5 the flux dependence of dispersive shifts $\chi_{1}$ and $\chi_{2}$, respectively. We see that away from resonances the shifts for set 2 are usually larger, as expected due to the larger coupling strengths (cf. Fig. 2). Moreover, due to the higher energy of

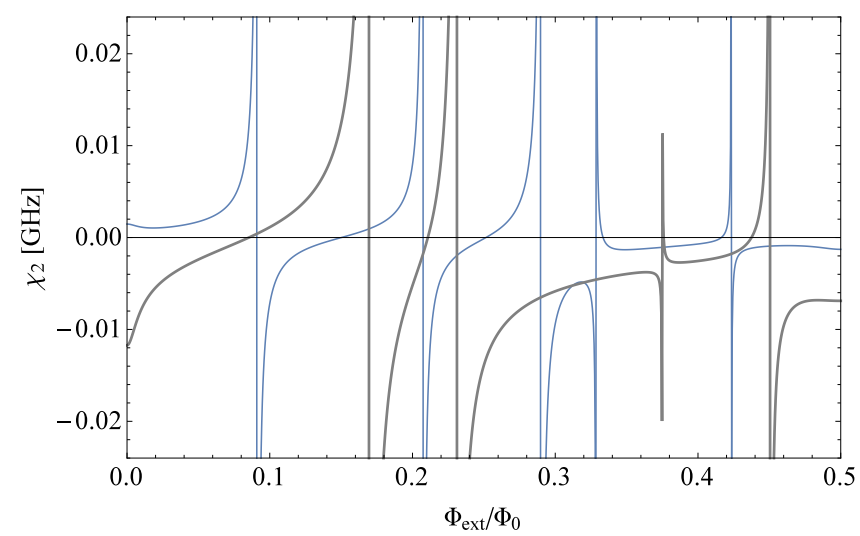

FIG. 5. (Color online) Dispersive shift $\chi_{2}$ [Eq. (36)] as function of external flux for parameter sets 1 (thin line) and 2 (thick line). The vertical scale covers a range (in absolute value) approximately one order of magnitude smaller than the coupling constant $g_{2}$ (cf. Fig. 2). This range is well within the validity of the dispersive regime: since higher-order corrections neglected here are suppressed by a factor of $\operatorname{order}\left(\chi_{2} / g_{2}\right)^{2}$ [34], appreciable deviations from the calculated values are possible only close to the resonances. 


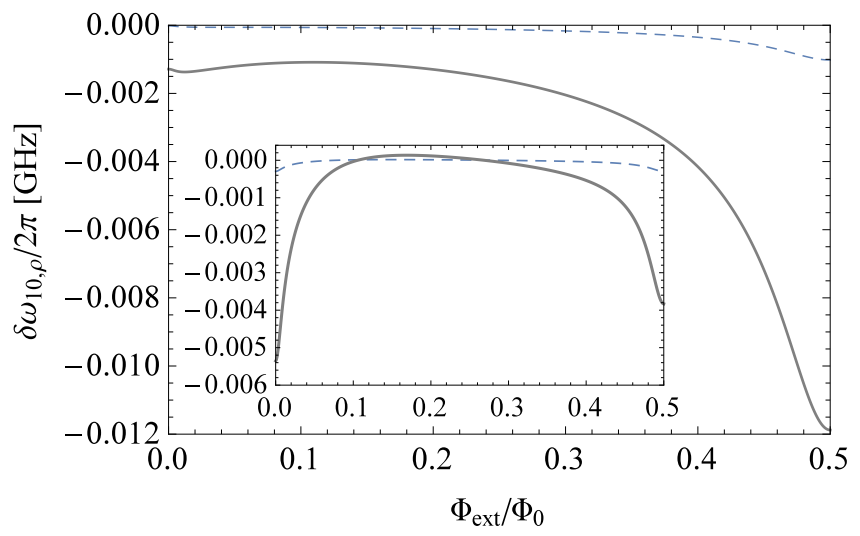

FIG. 6. (Color online) Contributions $\delta \omega_{10,1}$ (thick line) and $\delta \omega_{10,2}$ (thin, dashed line) [Eq. (38)] to the qubit frequency renormalization $\delta \omega_{10}$ for parameter sets 1 (inset) and 2 (main panel). For comparison, we note that the unrenormalized frequency $\omega_{10}$ approximately varies between $9 \mathrm{GHz}(8.2 \mathrm{GHz})$ at zero flux and $0.33 \mathrm{GHz}(0.64 \mathrm{GHz})$ at half-flux quantum for parameter set 1 (set 2).

the collective mode involved, the shifts $\chi_{2}$ displays a richer resonance structure. We will comment on the effect of these shifts on qubit coherence in Sec. VIII. Next, we consider the effect of the qubit-mode coupling on the qubit frequency in the absence of excitations of the modes.

\section{B. Qubit frequency renormalization}

In the preceding section, we studied the change in the qubit frequency when a collective mode is excited, but the interaction term $H_{\text {int }}$ of Eq. (32) modifies the qubit frequency even in the absence of collective mode excitations $\omega_{10} \rightarrow \omega_{10}+\delta \omega_{10}$. This frequency correction $\delta \omega_{10}$, arising from Lamb-type energy level shifts, is given by

$$
\delta \omega_{10}=\sum_{\rho} \delta \omega_{10, \rho}
$$

with

$$
\begin{aligned}
\delta \omega_{10, \rho}= & \frac{1}{2} \sqrt{\frac{E_{J}^{a}}{8 E_{C, \rho}^{e}}} g_{\rho}^{2}\left[\left|\left\langle 0\left|p_{\phi}\right| 1\right\rangle\right|^{2} \frac{2 \omega_{10}}{\omega_{10}^{2}-\omega_{\rho}^{2}}\right. \\
& +\sum_{l \geqslant 2}\left|\left\langle 0\left|p_{\phi}\right| l\right\rangle\right|^{2} \frac{1}{\omega_{l 0}+\omega_{\rho}} \\
& \left.-\sum_{l \geqslant 2}\left|\left\langle 1\left|p_{\phi}\right| l\right\rangle\right|^{2} \frac{1}{\omega_{l 1}+\omega_{\rho}}\right] .
\end{aligned}
$$

While this formula resembles Eq. (36), there is one important difference: so long as $\omega_{\rho}>\omega_{10}$, there are no divergences in Eq. (38). In Fig. 6, we plot the first two largest contributions to $\delta \omega_{10}$, namely, $\delta \omega_{10,1}$ and $\delta \omega_{10,2}$. The former is generally much larger (in absolute value) than the latter, due to the stronger coupling (cf. Fig. 2). Note that even at half-flux quantum, where $\omega_{10}$ has a minimum $[10,11]$, the correction is at most a few percent of $\omega_{10}$.

\section{Purcell rate}

So far, we have considered the system to be capacitively coupled to external voltage sources. In practical realizations of circuit QED experiments, this coupling is to a mode of a cavity; this can be accounted for by replacing [35]

$$
V \rightarrow \tilde{V}\left(c^{\dagger}+c\right)
$$

in the coupling Hamiltonian $H_{V}$ [Eq. (33)]. Here, parameter $\tilde{V}$ accounts for the strength of the electric field at the qubit position as well as for the geometry of the cavity-qubit system, while $c^{\dagger}(c)$ are creation (annihilation) operators for photons in the cavity. As it is customary, to include the cavity and its coupling to an external bath of harmonic oscillators, we add to $H_{e}$ in Eq. (29) the following Hamiltonian $H_{c b}$ :

$$
H_{c b}=\omega_{c} c^{\dagger} c+\sum_{k} \omega_{k} b_{k}^{\dagger} b_{k}+\sum_{k} \lambda_{k}\left(b_{k}^{\dagger} c+c^{\dagger} b_{k}\right),
$$

where $\omega_{c}$ is the cavity frequency, $b_{k}^{\dagger}\left(b_{k}\right)$ are creation (annihilation) operators for bath excitations with energy $\omega_{k}$, and $\lambda_{k}$ the coupling strengths between cavity and bath modes.

Within this model, one can calculate using Fermi's golden rule the so-called Purcell rate $\kappa_{q}$ for the qubit; that is, the decay rate of the qubit excited state by emission of a photon into the bath (mediated by the cavity)

$$
\kappa_{q}(f)=\kappa \frac{g_{q}^{2}(f)}{\left[\omega_{10}(f)-\omega_{c}\right]^{2}},
$$

where $\kappa$ is the inverse lifetime of a photon in the cavity, as determined by the cavity-bath couplings $\lambda_{k}$ [35], and

$$
g_{q}=2 e \tilde{V} \frac{\tilde{E}_{C}^{\phi}}{E_{C}^{c}}\left\langle 0\left|p_{\phi}\right| 1\right\rangle .
$$

This coupling constant depends on flux via the qubit states. The above expression for $\kappa_{q}$ is valid in the dispersive regime $g_{q} \ll\left|\omega_{10}-\omega_{c}\right|$. The similar calculation for the collective modes gives their decay rate as

$$
\kappa_{\rho}=\kappa \frac{1}{\left(\omega_{\rho}-\omega_{c}\right)^{2}}\left[\frac{e \tilde{V}}{4 E_{C}^{c}} g_{\rho} \frac{1}{\sqrt{2} \ell_{\rho}}\right]^{2},
$$

where $\ell_{\rho}=\left(8 E_{C, \rho}^{e} / E_{J}^{a}\right)^{1 / 4}$ and again we have assumed that the factor multiplying $\kappa$ is small compared to unity. Note that the term in square brackets is a decreasing function of $\rho$; therefore, so long as all the modes have frequency above the cavity one $\left(\omega_{1}>\omega_{c}\right)$, the decay rate of the collective modes decreases with $\rho$. If the qubit lifetime is limited by Purcell relaxation, we can then estimate the modes' lifetimes by eliminating the unknown quantity $\tilde{V}$ from Eqs. (41) and (43). In fact, the ratio $\kappa_{\rho} / \kappa_{q}(f)$ is also independent of $\kappa$, and it is determined by the circuit properties and the cavity frequency; we plot $\kappa_{\rho} / \kappa_{q}(0)$ in Fig. 7 for the parameters given in Table I, with $\omega_{c} / 2 \pi=8.18 \mathrm{GHz}$ for set 1 and $\omega_{c} / 2 \pi=8.89 \mathrm{GHz}$ for set 2 (see Refs. $[9,11]$, respectively).

\section{NONLINEARITY OF THE ARRAY JUNCTIONS}

By expanding the last term in Eq. (7) to quadratic order to obtain the approximate potential energy term in Eq. (10), we have treated the Josephson junctions in the array as 


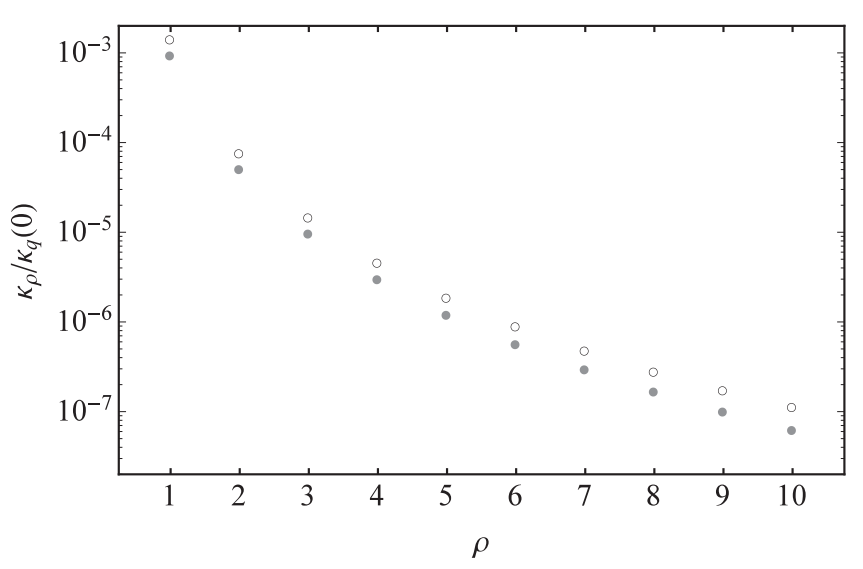

FIG. 7. Ratio $\kappa_{\rho} / \kappa_{q}(0)$ between collective mode and qubit Purcell decay rates [Eqs. (43) and (41), respectively] for the 10 lowest even modes. Filled circles: parameter set 1 in Table I; empty circles: set 2.

linear elements. However, the cosine in Eq. (7) includes their nonlinear properties, and in this section we account perturbatively for these nonlinearities. To begin with, we split the Josephson energy of each junction into two contributions using the identity

$$
\begin{aligned}
\cos \left[\frac{\phi}{N}+\sum_{\mu} W_{\mu m} \xi_{\mu}\right]= & \cos \frac{\phi}{N} \cos \left[\sum_{\mu} W_{\mu m} \xi_{\mu}\right] \\
& -\sin \frac{\phi}{N} \sin \left[\sum_{\mu} W_{\mu m} \xi_{\mu}\right]
\end{aligned}
$$

The sine product term, as we discuss in the following, generates two qubit-collective mode interaction terms that we will denote with $U_{\phi \xi}^{(1)}$ and $U_{\phi \xi}^{(3)}$. The product of the two cosines can be rewritten as

$$
\begin{aligned}
& \cos \frac{\phi}{N} \cos \left[\sum_{\mu} W_{\mu m} \xi_{\mu}\right] \\
& =\cos \frac{\phi}{N} \overline{\mathcal{C}}_{0 m}+\cos \left[\sum_{\mu} W_{\mu m} \xi_{\mu}\right]-\overline{\mathcal{C}}_{0 m} \\
& +\left(\cos \frac{\phi}{N}-1\right)\left(\cos \left[\sum_{\mu} W_{\mu m} \xi_{\mu}\right]-\overline{\mathcal{C}}_{0 m}\right),
\end{aligned}
$$

where

$$
\overline{\mathcal{C}}_{0 m}=\left\langle\cos \left[\sum_{\mu} W_{\mu m} \xi_{\mu}\right]\right\rangle_{0}
$$

is the expectation value of the operator inside the angular brackets in the ground state of the collective modes. As detailed in the next section, the first term in the right-hand side of Eq. (45) gives rise to the qubit mode effective potential $U_{\phi}$, while the last term is a qubit-collective modes interaction contribution, denoted with $U_{\phi \xi}^{(2)}$. The third term is a constant that can be neglected. The second term gives, to lowest order, the harmonic potential energy of the collective modes,

$$
\sum_{m} \cos \left[\sum_{\mu} W_{\mu m} \xi_{\mu}\right] \approx N-\frac{1}{2} \sum_{\mu} \xi_{\mu}^{2}+\cdots
$$

The higher-order terms in the expansion neglected here lead to interactions among the collective modes that do not affect the qubit directly. We do not consider such interactions from now on, and write the potential energy $U_{S}$ in the approximate form

$$
U_{S} \simeq \frac{1}{2} E_{J}^{a} \sum_{\mu} \xi_{\mu}^{2}+U_{\phi}+\sum_{j=1}^{3} U_{\phi \xi}^{(j)}
$$

with the potentials $U_{\phi}$ and $U_{\phi \xi}^{(j)}$ specified in what follows.

\section{A. Qubit effective potential $\boldsymbol{U}_{\boldsymbol{\phi}}$}

Keeping only the first term in the right-hand side of Eq. (45), from Eq. (7) we find

$$
U_{\phi}=-E_{J}^{b} \cos \left(\phi+\varphi_{e}\right)-E_{L}\left[N^{2} \cos \frac{\phi}{N}\right] \overline{\mathcal{C}}_{0}
$$

with $\overline{\mathcal{C}}_{0}=\sum_{m} \overline{\mathcal{C}}_{0 m} / N$. Upon expansion of the term in square brackets (valid for $|\phi| \ll \pi N$ ) and assuming $\overline{\mathcal{C}}_{0} \approx 1$, we recover the quadratic inductive energy term $E_{L} \phi^{2} / 2$ of Eq. (10). The full qubit potential, however, contains small additional nonlinearities originating from the higher-order terms of the expansion. Here, we do not consider these terms further, as they are suppressed by factors of the form $1 / N^{2 j}(j=1,2,3, \ldots)$, but we show that in general $\overline{\mathcal{C}}_{0}<1$; therefore, the actual inductive energy

$$
\tilde{E}_{L}=E_{L} \overline{\mathcal{C}}_{0}
$$

is smaller than what the simple expression $E_{L}=E_{J}^{a} / N$ suggests.

The expectation value entering $\overline{\mathcal{C}}_{0 m}$ [Eq. (46)] can be readily obtained from the known matrix elements for the harmonic oscillator (see, for example, Appendix D of [25]). Here, we have to remember that in the odd sector a rotation from the original modes $\zeta_{\rho}$ to independent modes $\tilde{\zeta}_{\rho}$ is necessary (see Appendix $\mathrm{C}$ ); this is accomplished via the orthogonal matrix $\Lambda_{\mu \nu}$ defined in Eq. (C12). We thus arrive at

$$
\overline{\mathcal{C}}_{0}=\frac{1}{N} \sum_{m} \exp \left[-\frac{1}{4} \sum_{\mu, v, \tau} W_{\mu m} W_{\nu m} \Lambda_{\tau \mu} \Lambda_{\tau \nu} \ell_{\tau}^{2}\right],
$$

where

$$
\ell_{\tau}=\left(8 E_{C, \tau} / E_{J}^{a}\right)^{1 / 4}
$$

is the oscillator length for mode $\tau$, with $E_{C, \tau}$ given in Eqs. (C10) and (24) for odd and even modes, respectively. Clearly, $\overline{\mathcal{C}}_{0}<1$ so long as at least one oscillator length is finite. We can also find a lower bound (and rough estimate) for $\overline{\mathcal{C}}_{0}$ by noting that $\ell_{\tau} \leqslant \ell_{0}$, where $\ell_{0}=\left(8 E_{C}^{a} / E_{J}^{a}\right)^{1 / 4}$ is the oscillator length in the absence of capacitance to ground in the array; note that we typically have $\ell_{0} \lesssim 1$ (cf. Table I). Then, using the identities $\sum_{\tau} \Lambda_{\tau \nu}^{2}=1$ and $\sum_{\mu} W_{\mu m}^{2}=(N-1) / N$, 
we find

$$
\overline{\mathcal{C}}_{0} \geqslant \exp \left[-\ell_{0}^{2} \frac{N-1}{4 N}\right] .
$$

The expansion to lowest order in $\ell_{0}^{2}$ of this formula agrees with the expression for the reduction of $E_{L}$ reported in Ref. [24]. Our result shows that that expression generally overestimates the suppression of the inductive energy.

\section{B. Quadratic interaction $U_{\phi \xi}^{(2)}$}

We now consider the leading-order contribution to the potential energy originating from the last term in Eq. (45). By expanding the term dependent on the collective mode coordinates and introducing the creation/annhilation operators via $\xi_{\mu}=\ell_{\mu}\left(a_{\mu}+a_{\mu}^{\dagger}\right) / \sqrt{2}$ we arrive at

$$
U_{\phi \xi}^{(2)}=\frac{E_{J}^{a}}{2}\left(1-\cos \frac{\phi}{N}\right) \sum_{\mu} \frac{\ell_{\mu}^{2}}{2}\left(2 a_{\mu}^{\dagger} a_{\mu}+a_{\mu}^{\dagger} a_{\mu}^{\dagger}+a_{\mu} a_{\mu}\right) .
$$

Note that in the absence of the array ground capacitances (so that $\ell_{\mu} \equiv \ell_{0}$ ) this interaction term is invariant under orthogonal transformations belonging to the group $\mathrm{O}(N-1)$; the $\mathrm{U}(N-$ 1) symmetry of approximate Lagrangian $\mathcal{L}_{U}$ in Eq. (11) is only partially broken [the first term in the sum actually fully preserves $\mathrm{U}(N-1)$ symmetry].

In Eq. (54), we can distinguish two contributions. First, there are terms which are proportional to each collective modes number operator $n_{\mu}=a_{\mu}^{\dagger} a_{\mu}$; to lowest order in $1 / N$ these terms are

$$
U_{\delta E_{L}}=\frac{1}{2} \phi^{2}\left[\frac{E_{L}}{2 N} \sum_{\mu} \ell_{\mu}^{2} a_{\mu}^{\dagger} a_{\mu}\right],
$$

and they give a dependence of the inductive energy $E_{L}$ on the occupation of the collective modes. Since changes in $E_{L}$ lead to variations of the qubit frequency, this dependence can be interpreted as a dispersive shift $\chi_{\mu}^{\delta E_{L}}$ :

$$
\chi_{\mu}^{\delta E_{L}}=\frac{1}{2}\left(\frac{\partial \omega_{10}}{\partial E_{L}}\right) \frac{E_{L}}{2 N} \ell_{\mu}^{2} .
$$

Over a broad range of fluxes, except near half-integer multiples of the flux quantum, the qubit frequency is approximately proportional to the inductive energy [25]

$$
\omega_{10} \approx(2 \pi)^{2} E_{L}\left|f-\frac{1}{2}\right|,
$$

so that $E_{L}\left(\partial \omega_{10} / \partial E_{L}\right) \approx \omega_{10}$. This approximate relation translates at all fluxes in an upper bound for the dispersive shifts:

$$
\chi_{\mu}^{\delta E_{L}} \lesssim \frac{\omega_{10}}{4 N} \ell_{\mu}^{2}
$$

This bound shows that the dispersive shifts lead to relative changes of order $1 / N$, in the qubit frequency. Interestingly, the derivative $\partial \omega_{10} / \partial E_{L}$ and hence the dispersive shifts have minima at half-integer multiples of the flux quantum (see Fig. 8). Therefore, the dephasing induced by $U_{\delta E_{L}}$ is suppressed at these "sweet spots," similar to the suppression of dephasing by flux noise; this is not surprising since at

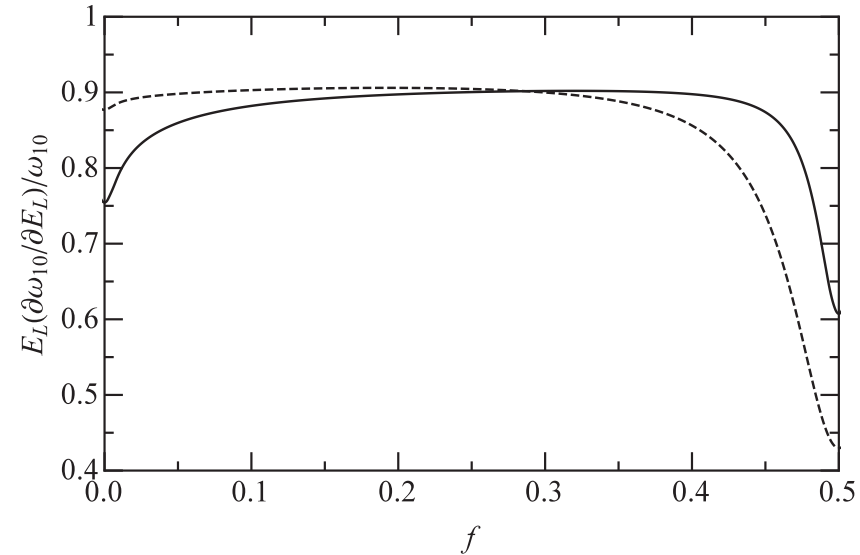

FIG. 8. Normalized derivative $\partial \omega_{10} / \partial E_{L}$ calculated for the parameters in Table I. Solid line: set 1; dashed line: set 2 . Note the minima at $f=0$ and $\frac{1}{2}$.

leading order the flux and inductive energy affect the qubit frequency in the same way [see Eq. (57)]. However, for typical experimental parameters, the suppression is less than one order of magnitude.

The second type of contribution in $U_{\phi \xi}^{(2)}$ comes from terms of the form $a_{\mu} a_{\mu}+a_{\mu}^{\dagger} a_{\mu}^{\dagger}$. As in Sec. IV, the effect of such terms can be studied by performing a Schrieffer-Wolff transformation, as detailed in Appendix E. Here, we simply note that, since they involve the virtual exchange of two collective mode excitations rather than one, the resulting dispersive shifts are generally smaller than $\chi_{\mu}^{\delta E_{L}}$ of Eq. (56) and can therefore be neglected.

\section{Linear interaction $U_{\phi \xi}^{(1)}$}

In this section and the next one, we focus on the perturbative treatment of the last term in Eq. (44), obtained by expanding the sine with argument the collective modes coordinates. The contribution to the potential energy from the linear term in this expansion vanishes by construction, due to the property $\sum_{m} W_{\mu m}=0$. As we show in Appendix G, the third-order term gives rise to two types of interactions, one of them being a linear interaction term of the form

$$
\begin{aligned}
U_{\phi \xi}^{(1)} & =\sum_{\rho=1}^{N_{e}} \tilde{g}_{\rho}\left(N \sin \frac{\phi}{N}\right) \eta_{\rho}, \\
\tilde{g}_{\rho} & =\frac{1}{2(2 N)^{3 / 2}} E_{J}^{a}\left[\ell_{N-\rho}^{2}-\ell_{\rho}^{2}\right]
\end{aligned}
$$

with $\eta_{\rho}$ and $N_{e}$ defined in the text after Eq. (23). Since it couples the qubit mode with even collective modes only, this interaction preserves $P T$ symmetry. Moreover, in the $\mathrm{S}_{N}$ symmetric case (i.e., neglecting ground capacitances, so that $\ell_{\mu} \equiv \ell_{0}$ for all $\mu$ ) this term is absent, in agreement with the group-theoretical analysis of [24]. The coupling constant $\tilde{g}_{\rho}$ decreases with the collective mode index $\rho$, albeit more slowly than $g_{\rho}$ in Eq. (32) for low $\rho$, while for large index $\rho \lesssim N_{e}$ we find

$$
\frac{\tilde{g}_{\rho}}{g_{\rho}} \approx \frac{\omega_{p}^{a}}{32 N \tilde{E}_{C}^{\phi}}
$$




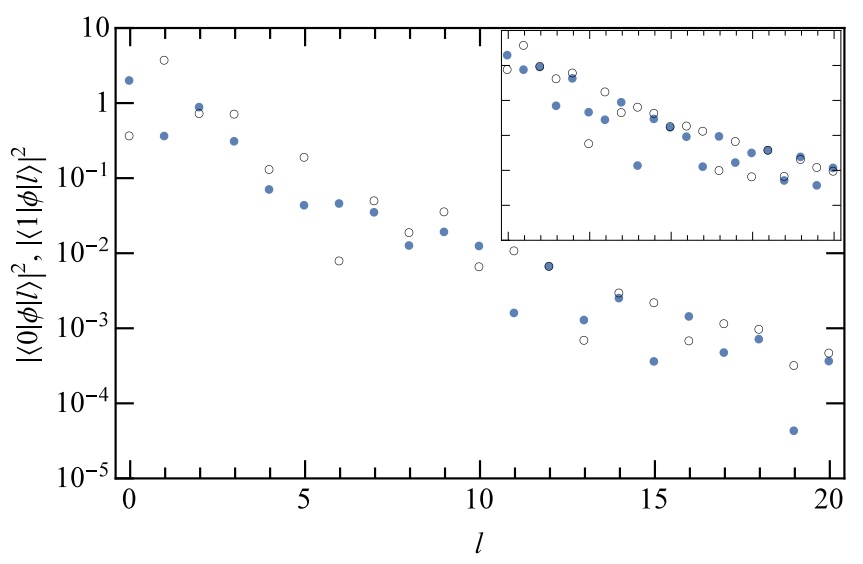

FIG. 9. (Color online) Main panel: matrix elements squared $|\langle 0|\phi| l\rangle|^{2}$ (filled circles) and $|\langle 1|\phi| l\rangle|^{2}$ (empty circles) at $f=0.35$ for $l \leqslant 20$ calculated using parameter set 1 (see Table I). Inset: same as main panel but for parameter set 2 .

where $\omega_{p}^{a}=\sqrt{8 E_{J}^{a} E_{C}^{a}}$ is the array junction plasma frequency. A (loose) upper bound for $\tilde{g}_{1}$ is given by

$$
\tilde{g}_{1}<\frac{E_{J}^{a}}{2} \frac{\ell_{0}^{2}}{(2 N)^{3 / 2}}=\frac{1}{2} \frac{\omega_{p}^{a}}{(2 N)^{3 / 2}} .
$$

As done for the interaction term $H_{\text {int }}$ in Eq. (32), the effect of $U_{\phi \xi}^{(1)}$ on the qubit can be more easily studied by performing a Schrieffer-Wolff transformation leading to the additional dispersive shift $\chi_{\rho}^{(1)}(f)$ :

$$
\begin{aligned}
\chi_{\rho}^{(1)}= & \frac{1}{2} \sqrt{\frac{8 E_{C, \rho}^{e}}{E_{J}^{a}}} \tilde{g}_{\rho}^{2}\left[\left|\left\langle 0\left|\left(N \sin \frac{\phi}{N}\right)\right| 1\right\rangle\right|^{2} \frac{2 \omega_{10}}{\omega_{10}^{2}-\omega_{\rho}^{2}}\right. \\
& +\sum_{l \geqslant 2}\left|\left\langle 0\left|\left(N \sin \frac{\phi}{N}\right)\right| l\right\rangle\right|^{2} \frac{\omega_{l 0}}{\omega_{l 0}^{2}-\omega_{\rho}^{2}} \\
& \left.-\sum_{l \geqslant 2}\left|\left\langle 1\left|\left(N \sin \frac{\phi}{N}\right)\right| l\right\rangle\right|^{2} \frac{\omega_{l 1}}{\omega_{l 1}^{2}-\omega_{\rho}^{2}}\right]
\end{aligned}
$$

A few comments are in order: first, since the low-lying states are localized (so that relevant values of $\phi$ are at most of order $2 \pi$ ) and $N$ is large, a good approximation for the matrix elements is obtained with the substitution $N \sin \phi / N \rightarrow \phi$. Second, within this approximation, numerical calculation of $\chi_{\rho}^{(1)}$ does not require much additional computation compared to that of $\chi_{\rho}$ [Eq. (36)], thanks to the identity [37]

$$
\omega_{m l}^{2}|\langle m|\phi| l\rangle|^{2}=\left(8 \tilde{E}_{C}^{\phi}\right)^{2}\left|\left\langle m\left|p_{\phi}\right| l\right\rangle\right|^{2} .
$$

Third, the pole structure in Eq. (62) is the same as that of $\chi_{\rho}$ and the matrix elements again become smaller as $l$ increases [see Fig. 9 and Eq. (63)]. Finally, for typical experimental parameters, the coupling constants $\tilde{g}_{\rho}$ are at least two orders of magnitude smaller than $g_{\rho}$ [cf. Fig. 10], so we expect the dispersive shifts $\chi_{\rho}^{(1)}$ to have negligible effect. More precisely, for almost all values of flux [38] we have $\left|\chi_{\rho}^{(1)} / \chi_{\rho}\right| \sim\left(\tilde{g}_{\rho} / g_{\rho}\right)^{2}$ and the latter quantity is $\lesssim 10^{-5}$ for experimentally relevant parameters; therefore, we neglect $\chi_{\rho}^{(1)}$ from now on.

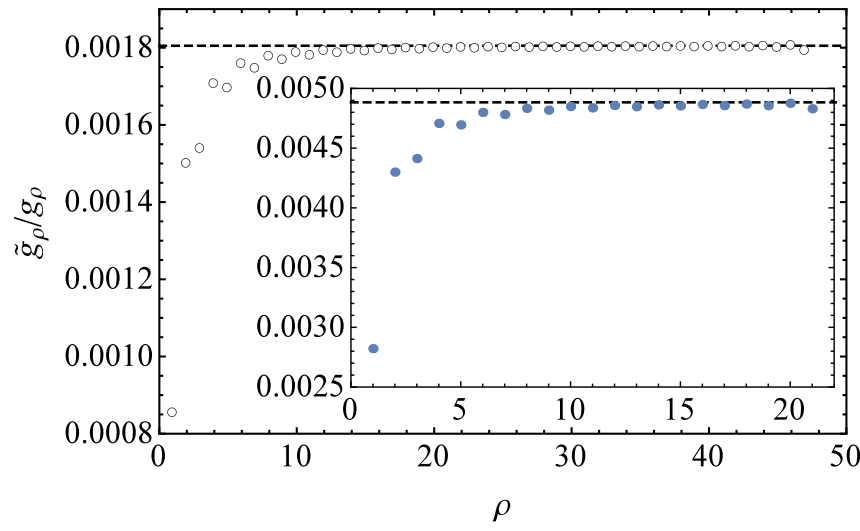

FIG. 10. (Color online) Coupling constants ratio $\tilde{g}_{\rho} / g_{\rho}$ for parameter set 1 (inset, filled circles) and 2 (main panel, empty circles) for all the even modes. Horizontal dashed lines are given by the right-hand side of Eq. (60).

\section{Multimode interaction $U_{\phi \xi}^{(3)}$}

All the interactions discussed so far involve the qubit mode and a single collective mode. This is not the case for the interaction term $U_{\phi \xi}^{(3)}$, which involves up to three collective modes:

$$
\begin{aligned}
U_{\phi \xi}^{(3)}= & -\frac{E_{J}^{a}}{24 \sqrt{N}} \sin \frac{\phi}{N}\left[3 \sum_{\mu, v=1}^{\mu+v<N} \ell_{\mu} \ell_{\nu} \ell_{\mu+\nu}\right. \\
& \times\left(a_{\mu+\nu}^{\dagger} a_{\nu}^{\dagger} a_{\mu}^{\dagger}+2 a_{\mu+\nu}^{\dagger} a_{\nu}^{\dagger} a_{\mu}+a_{\nu}^{\dagger} a_{\mu}^{\dagger} a_{\mu+\nu}+\text { H.c. }\right) \\
& -\sum_{\mu+\nu>N}^{N-1} \ell_{\mu} \ell_{\nu} \ell_{2 N-\mu-v}\left(a_{2 N-\mu-\nu}^{\dagger} a_{\nu}^{\dagger} a_{\mu}^{\dagger}\right. \\
& \left.\left.+3 a_{2 N-\mu-\nu}^{\dagger} a_{\nu}^{\dagger} a_{\mu}+\text { H.c. }\right)\right]
\end{aligned}
$$

where H.c. denotes the Hermitian conjugate. The first term, for example, contains creation operators of two modes if $\mu=$ $v$ and three modes if $\mu \neq v$. Note that the index structure ensures that at least one index is even and the remaining two indices have the same parity; this shows that $P T$ symmetry is preserved.

As a consequence of the presence of three creationannihilation operators in Eq. (64), a description in terms of an effective Hamiltonian would involve terms with products of up to three number operators (see also the discussion of the $U_{\phi \xi}^{(2)}$ interaction in Appendix E). Rather than attempting such a complicated description here, we consider the case in which the occupation probability of each mode is sufficiently small that we can neglect the possibility of having two or more excitations in a mode or two or more modes being excited at the same time; this requires the occupation probability to be small compared to $1 / N$ (see Appendix F). In other words, we only consider the possibility that no more than one collective mode is excited at any given time. We can then calculate the change $\Delta \omega_{10, \mu}$ in qubit frequency from when the collective modes are in their ground state $|0\rangle\left(a_{\mu}|0\rangle=0\right.$ for any $\left.\mu\right)$ to when one of the collective modes is excited, i.e., in state $\left|1_{\mu}\right\rangle=a_{\mu}^{\dagger}|0\rangle$. Such 
a frequency change resembles the dispersive shifts discussed so far, although those are valid for multiple excitations in each mode. The perturbative calculation of the frequency change is detailed in Appendix G; there we also show that the frequency change is smaller than the dispersive shift $\chi_{\mu}^{\delta E_{L}}$ in Eq. (56) and can therefore be neglected. We explore the effects of the collective modes dispersive shifts on the qubit in Sec. VIII, while in the next section we turn our attention to the case of a circuit asymmetrically coupled to the cavity.

\section{BROKEN PT SYMMETRY: AN EXAMPLE}

In all the previous sections we have assumed the system to be $P T$ symmetric, which ensures the decoupling of qubit and odd collective modes in the (approximate) quadratic Lagrangian. In practice, it is difficult to fabricate a perfectly symmetric circuit, so it is interesting to investigate what are the main qualitative consequences of breaking parity symmetry. To this end, we consider a simple case in which the symmetry is broken by taking the two coupling capacitors to have different values:

$$
C_{c}^{0}=C_{c}+C_{t} \delta c / 2, \quad C_{c}^{N}=C_{c}-C_{t} \delta c / 2
$$

with $C_{t}$ defined in Eq. (16). Therefore, in this section $C_{c}$ represent the average of the two coupling capacitors, and we have introduced the dimensionless asymmetry parameter

$$
\delta c=\frac{C_{c}^{0}-C_{c}^{N}}{C_{t}}
$$

with $|\delta c| \leqslant 1$. Inclusion of the asymmetric capacitive coupling in the circuit Lagrangian amounts to the substitutions [39]

$$
\begin{gathered}
G_{00} \rightarrow G_{00}-\frac{1}{4 E_{t}}(\delta c)^{2}, \\
G_{0 \mu} \rightarrow G_{0 \mu}+\frac{\delta c}{2 E_{g}^{a}} \frac{c_{\mu} o_{\mu}}{\sqrt{2 N} s_{\mu}^{2}}
\end{gathered}
$$

in Eqs. (13) and (14), and

$$
\mathcal{L}_{V} \rightarrow \mathcal{L}_{V}+\frac{(\delta c)^{2}}{8 E_{t}} \dot{\phi} e V-\frac{\delta c}{4 E_{g}^{a}} \sum_{\mu} \frac{c_{\mu} o_{\mu}}{\sqrt{2 N} s_{\mu}^{2}} \dot{\xi}_{\mu} e V
$$

in Eq. (21). Note that matrix $G_{\mu \nu}$ is not affected and, as discussed in Appendix $\mathrm{C}$, a rotation is needed to diagonalize it in the odd sector. This rotation in principle modifies the new term introduced in Eq. (68); we neglect this modification as it does not introduce any new qualitative feature; this is also a quantitatively good approximation if the parameter $\lambda$ [Eq. (17)] is sufficiently small.

Within the same approximations used previously [in particular, we assume again Eq. (28) to hold], the total Hamiltonian $H$ takes the same form as in Eq. (29):

$$
\begin{gathered}
H=\bar{H}_{\phi}+\sum_{\mu=1}^{N-1} H_{\mu}+H_{\mathrm{int}}+H_{V}, \\
\bar{H}_{\phi}=4 \bar{E}_{C}^{\phi} p_{\phi}^{2}-E_{J}^{b} \cos \left(\phi+\varphi_{e}\right)+\frac{1}{2} E_{L} \phi^{2}, \\
H_{\mu}=4 E_{C, \mu} p_{\mu}^{2}+\frac{1}{2} E_{J}^{a} \xi_{\mu}^{2},
\end{gathered}
$$

$$
\begin{gathered}
H_{\mathrm{int}}=\sum_{\mu=1}^{N-1} g_{\mu} p_{\mu} p_{\phi}, \\
H_{V}=-\sum_{\mu=1}^{N-1} g_{\mu} p_{\mu} e V\left[\frac{1}{4 E_{C}^{c}}-\frac{(\delta c)^{2}}{8 E_{t}}-\frac{o_{\mu}}{2 \bar{E}_{C}^{\phi}}\right] \\
-\bar{E}_{C}^{\phi} p_{\phi} e V\left[\frac{2}{E_{C}^{c}}-\frac{(\delta c)^{2}}{E_{t}}+\frac{(\delta c)^{2}}{2 N} \sum_{\mu} o_{\mu} \frac{E_{C, \mu}}{\left(E_{g}^{a}\right)^{2}} \frac{c_{\mu}^{2}}{s_{\mu}^{4}}\right] .
\end{gathered}
$$

Despite the formal similarity, there are important differences between Eqs. (29) and (70): first, all collective modes appear in $H$, not just the even ones; we remind here that $E_{C, \mu}$ is given by either Eqs. (24) or (C10) depending on the even/odd mode parity. Second, due to the asymmetry the qubit charging energy $\bar{E}_{C}^{\phi}$ is renormalized from the definition in Eq. (26):

$$
\frac{1}{\bar{E}_{C}^{\phi}}=\frac{1}{\tilde{E}_{C}^{\phi}}-\frac{(\delta c)^{2}}{4 E_{t}} .
$$

Third, the coupling constants $g_{\mu}$ are different for even and odd modes:

$$
g_{\mu}=\frac{4}{\sqrt{2 N}} \frac{\bar{E}_{C}^{\phi} E_{C, \mu}}{E_{g}^{a}} \frac{c_{\mu}}{s_{\mu}^{2}}\left(o_{\mu+1}-o_{\mu} \delta c\right) .
$$

The structure of Hamiltonian $H$ in Eq. (70) shows that the main consequence of breaking the parity symmetry is the introduction of coupling between qubit and odd modes with coupling strength linear in the asymmetry parameter $\delta c$. Therefore, for strong asymmetry $|\delta c| \sim 1$, the odd modes influence the qubit in the same way as the even ones. Even for moderate asymmetry $|\delta c| \sim 0.1$, the effect of the lower-energy odd modes may be non-negligible (at least near zero flux, where the qubit frequency is closer to those of the collective modes): while $\delta c$ suppresses the coupling of the odd modes to the qubit, the odd modes with index $2 \rho-1$ are closer in frequency to the qubit than the even modes with index $2 \rho$, and the smaller frequency difference generally increases the dispersive shift [see Eq. (36)]; also, the term proportional to $1 / \bar{E}_{C}^{\phi}$ in Eq. (74) roughly compensates for the $\delta c$ suppression of coupling between odd modes and cavity, thus giving similar lifetimes for odd $(2 \rho-1)$ and even $(2 \rho)$ modes. On the other hand, small asymmetry at the percent level, as usually present in nominally symmetric devices, implies that the odd modes can be safely neglected.

Asymmetrically coupled systems similar to that described above have been recently probed experimentally, which enable us to test in part our theory. For example, in Ref. [18] an array of 80 junctions was placed in parallel to a (resonator) capacitor and the 9 lowest resonant frequencies were measured. The system is described by the Hamiltonian $H$ in Eq. (70) if we set $E_{J}^{b}=0$. Then, $H$ describes a harmonic oscillator linearly coupled to $N-1$ oscillators. The resonant frequencies of the corresponding $N$-independent oscillators can be easily calculated numerically; to compare with experiments, we note that the lowest mode in the experiment corresponds to what we call the qubit mode $\phi$, and therefore the higher even indices 


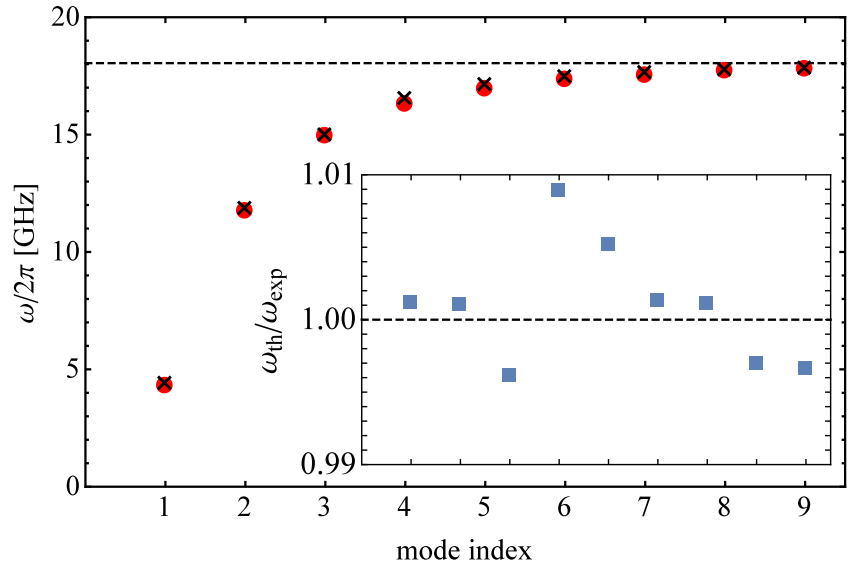

FIG. 11. (Color online) Comparison between collective modes frequencies measured in Ref. [18] (red circles) with those calculated using the present theory (black crosses). The inset shows the ratio between theoretical and experimental frequencies.

correspond to our odd modes and vice versa, odd mode indices in the experiments correspond to our even modes.

In calculating the resonant frequencies, we use as input parameters the array junction capacitance $C_{J}^{a}=40.1 \mathrm{fF}$ $\left(E_{C}^{a} \simeq 483 \mathrm{MHz}\right.$ ) and its Josephson inductance $L_{J}=1.94 \mathrm{nH}$ $\left(E_{J}^{a} \simeq 84.3 \mathrm{GHz}\right.$ ), the capacitance to ground $C_{g}^{a}=114 \mathrm{aF}$ $\left(E_{g}^{a} \simeq 170 \mathrm{GHz}\right.$ ), the two coupling capacitors $C_{c}^{0}=12.2$ $\mathrm{fF}$ and $C_{c}^{N}=5.1 \mathrm{fF}\left(E_{C}^{c} \simeq 2.24 \mathrm{GHz}, \delta c \simeq 0.27\right)$, and the resonator capacitor $C_{J}^{b}=3.19 \mathrm{fF}\left(E_{C}^{b} \simeq 6.07 \mathrm{GHz}\right)$. The frequencies calculated with these parameters differ by less than $1 \%$ from the measured frequencies (see Fig. 11). We note that while the array junction parameters $C_{J}^{a}$ and $L_{J}$ agree with those reported in Ref. [18], the ground capacitance $C_{g}^{a}$ we estimate here is about three times bigger. Since the calculations throughout the paper are based on the original estimate of [18], they could underestimate, e.g., the dispersive shifts and the decay rates by almost one order of magnitude; we will return to this point when estimating the dephasing rate in Sec. VIII.

A very recent experiment [40] reports the measurement of 14 resonant frequencies in an array of 200 junctions without shunting capacitor $\left(C_{J}^{b}=0\right)$. We can again compare our calculated frequencies with the measured ones: setting $C_{g}^{a}=98 \mathrm{aF}$ and optimizing the other parameters, we find again differences of less than $1 \%$ except for the third mode, whose measured frequency is about $7 \%$ higher than the calculated one; this larger difference is likely due to the presence near the frequency of that mode of a spurious resonance [40].

\section{COUPLING INTO THE SUPERINDUCTANCE}

So far, both for the $P T$-symmetric and the brokensymmetry cases, we have taken the coupling capacitors to be connected to the two islands separated by the phase-slip junction. The coupling capacitors can be attached to any island in the circuit, and in fact such a setup has been used in more recent experiments [11,41]. In general, arbitrary placement will immediately break parity symmetry even if the capacitances are the same for both capacitors. Here, we consider briefly the simplest case of equal capacitors placed

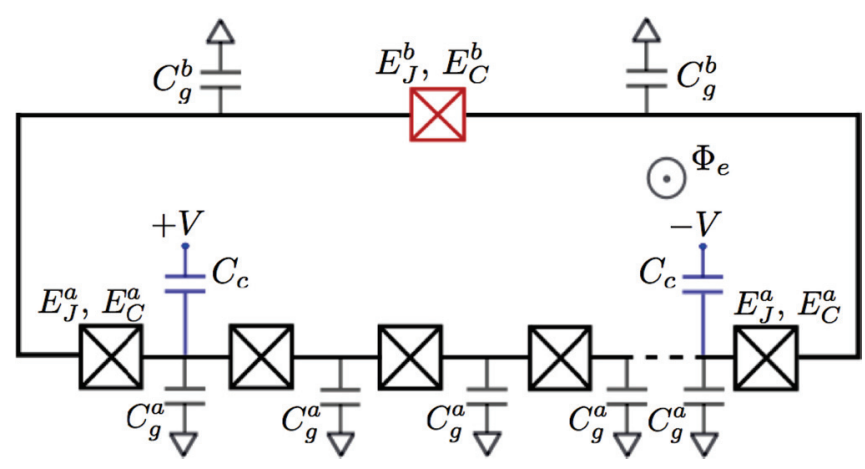

FIG. 12. (Color online) Circuit model for the fluxonium with coupling capacitors attached to islands 1 and $N-1$.

symmetrically with respect to the phase-slip junction, so that parity symmetry is preserved and no qubit-odd mode interaction is allowed. Concretely, we take the first capacitor to be connected to island $\delta \geqslant 0$, where islands 0 and $N$ are the two islands surrounding the phase-slip junction; then, the second capacitor is connected to island $N-\delta$, and the maximum possible $\delta$ is $\delta_{M}=\lfloor(N-1) / 2\rfloor$. As an example, the circuit with $\delta=1$ is depicted in Fig. 12 .

With the coupling capacitors in this symmetric configuration, the coupling Lagrangian $\mathcal{L}_{V}$ [cf. Eq. (21)] becomes

$$
\mathcal{L}_{V}=-\frac{1}{4 E_{C}^{c}}\left(1-\frac{2 \delta}{N}\right) \dot{\phi} e V+\frac{1}{4 E_{C}^{c}} \sqrt{\frac{2}{N}} \sum_{\rho=1}^{N_{e}} \frac{s_{4 \delta \rho}}{s_{2 \rho}} \dot{\eta}_{\rho} e V .
$$

This formula correctly reduces to Eq. (21) for $\delta=0$, while for $\delta>0$ a new coupling between cavity and even modes is present.

Changing the position of the coupling capacitors also affects the kinetic energy part $T_{G}$ of the Lagrangian [cf. Eq. (12)], and a general treatment of this modified term is quite cumbersome. Here, we consider the simple limit in which we neglect the ground capacitances $C_{g}^{a}, C_{g}^{b} \rightarrow 0$. In this case, as we show in Appendix $\mathrm{H}$, the Hamiltonian is

$$
\begin{gathered}
H=\hat{H}_{\phi}+H_{2}+H_{\mathrm{int}}+H_{V}+\sum_{\mu \neq 2} H_{\mu}, \\
\hat{H}_{\phi}=4 \frac{\hat{E}_{C}^{\phi}}{1-\mathrm{g}^{2}} p_{\phi}^{2}-E_{J}^{b} \cos \left(\phi+\varphi_{e}\right)+\frac{1}{2} E_{L} \phi^{2}, \\
H_{2}=4 \frac{E_{C 2}}{1-\mathrm{g}^{2}} p_{2}^{2}+\frac{1}{2} E_{J}^{a} \xi_{2}^{2}, \\
H_{\mathrm{int}}=\frac{\mathrm{g} \sqrt{\hat{E}_{C}^{\phi} E_{C 2}}}{1-\mathrm{g}^{2}} p_{2} p_{\phi} \equiv \mathrm{g}_{2} p_{2} p_{\phi}, \\
H_{V}=-\frac{2 p_{\phi} e V}{1-\mathrm{g}^{2}} \frac{\hat{E}_{C}^{\phi}}{E_{C}^{c}}\left(1-\frac{2 \delta}{N}\right)\left[1-\delta\left(1-\frac{2 \delta}{N}\right) \frac{E_{C 2}}{E_{C}^{c}}\right] \\
\left.+\frac{4 p_{2} e V}{1-\mathrm{g}^{2}} \frac{E_{C 2}}{E_{C}^{c}} \sqrt{\delta\left(1-\frac{2 \delta}{N}\right.}\right)\left[1-\left(1-\frac{2 \delta}{N}\right)^{2} \frac{\hat{E}_{C}^{\phi}}{2 E_{C}^{c}}\right] \\
\equiv-\mathrm{g}_{\phi, c} p_{\phi} e V+\mathrm{g}_{2, c} p_{2} e V .
\end{gathered}
$$



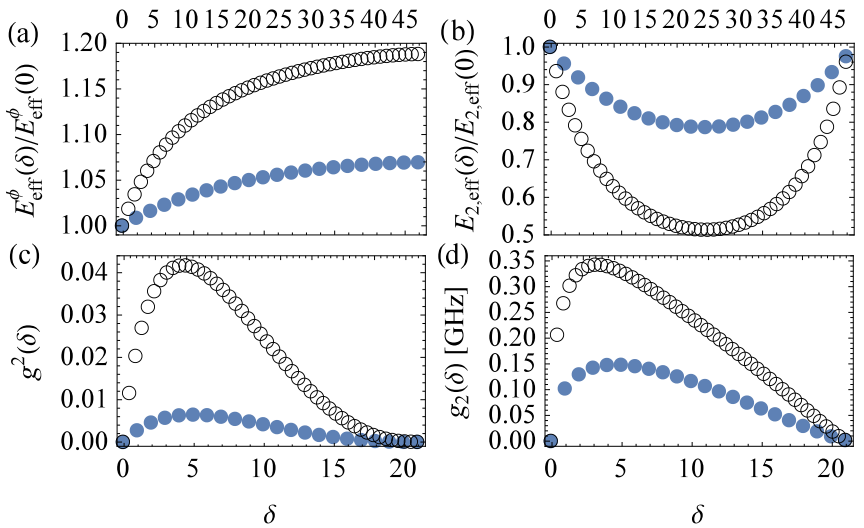

FIG. 13. (Color online) In all four panels, filled circles are used to denote parameter set 1 and empty ones for set 2 . The horizontal bottom (top) scales are used for set 1 (2). (a) Normalized effective qubit charging energy $E_{\mathrm{eff}}^{\phi}(\delta) / E_{\mathrm{eff}}^{\phi}(0)$ vs coupling capacitor position $\delta$. (b) Normalized collective mode charging energy $E_{2, \text { eff }}(\delta) / E_{2, \text { eff }}(0)$ vs $\delta$. (c) Dimensionless parameter g [Eq. (86)] vs $\delta$. (d) Qubit-collective mode coupling $\mathrm{g}_{2}$ [Eq. (82)] vs $\delta$.

The parameters $\mathrm{g}_{2}, \mathrm{~g}_{\phi, c}$, and $\mathrm{g}_{2, c}$ defined above, as well as the quantities

$$
\begin{aligned}
\frac{1}{\hat{E}_{C}^{\phi}} & =\frac{1}{E_{C}^{\phi}}+\frac{1}{2 E_{C}^{c}}\left(1-\frac{2 \delta}{N}\right)^{2}, \\
\frac{1}{E_{C 2}} & =\frac{1}{E_{C}^{a}}+\frac{1}{E_{C}^{c}} \delta\left(1-\frac{2 \delta}{N}\right), \\
\mathrm{g}^{2} & =\frac{\hat{E}_{C}^{\phi} E_{C 2}}{2\left(E_{C}^{c}\right)^{2}} \delta\left(1-\frac{2 \delta}{N}\right)^{3},
\end{aligned}
$$

depend on the position $\delta$ of the coupling capacitors.

The main qualitative feature of the Hamiltonian in Eq. (78) is that the qubit and the cavity both couple to only one collective mode whose charging energy is renormalized below $E_{C}^{a}$, while all the other $N-2$ modes remain degenerate and uncoupled. (Of course, in the presence of ground capacitances the degeneracy is lifted and all the even modes couple to both qubit and cavity.) While for typical experimental parameters the effective qubit charging energy $E_{\text {eff }}^{\phi}=\hat{E}_{C}^{\phi} /\left(1-\mathrm{g}^{2}\right)$ moderately increases as $\delta$ increases towards $N / 2$, the collective mode effective charging energy $E_{2, \text { eff }}=E_{C 2} /\left(1-\mathrm{g}^{2}\right)$ can be more strongly suppressed when $\delta \sim N / 4$ [see Figs. 13(a) and 13(b)]. The dimensionless parameter $\mathrm{g}$ varies nonmonotonically as function of $\delta$ and is generally small [see Fig. 13(c)]. The qubit-collective mode coupling strength $\mathrm{g}_{2}$ also depends significantly on $\delta$ [see Fig. 13(d)]; note that the largest values of $\mathrm{g}_{2}$ at $\delta \sim N / 8$ are a significant fraction of (or comparable to) the coupling strengths between qubit and lowest collective modes calculated for $\delta=0$ but in the presence of ground capacitances (see Fig. 2). These observations imply that by appropriately placing additional capacitors in the array, both the collective modes spectrum and the coupling strength with the qubit can be controlled to some degree. In particular, to minimize the effects of the collective modes on the qubit the coupling capacitors should either be placed next to the phase-slip junction $(\delta=0)$, or opposite to it $\left(\delta=\delta_{M}\right)$, while

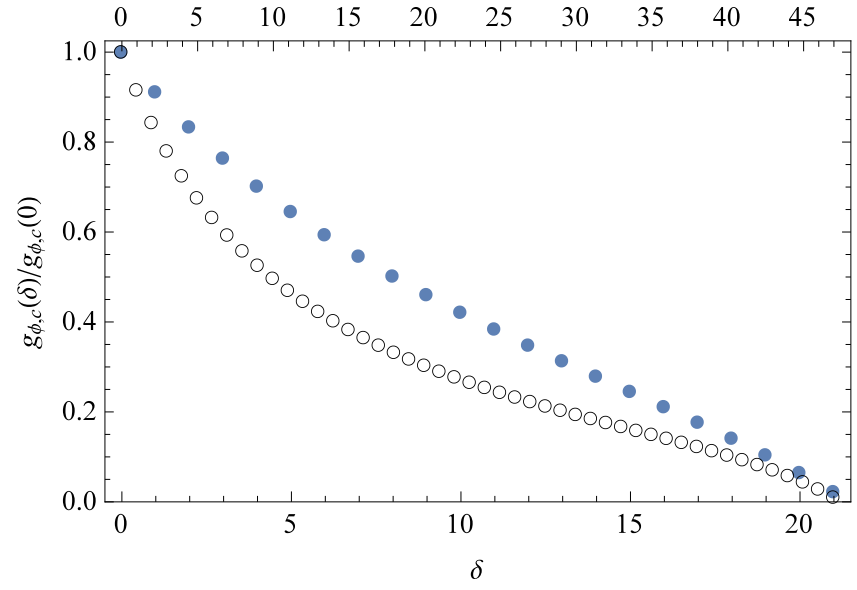

FIG. 14. (Color online) Normalized qubit-cavity coupling $\mathrm{g}_{\phi, c}$ [Eq. (83)] vs coupling capacitors position $\delta$. Horizontal bottom (top) scale is used for set 1 , filled circles (2, empty circles).

intermediate positions (especially in the range $\delta \sim N / 8-N / 4$ ) maximize those effects.

In Eq. (83), we give expressions for the qubit-cavity and collective mode-cavity dimensionless couplings $\mathrm{g}_{\phi, c}$ and $\mathrm{g}_{2, c}$. For $\delta=0, \mathrm{~g}_{\phi, c}$ reduces to the value in Eq. (33); as $\delta$ increases, $\mathrm{g}_{\phi, c}$ gradually decreases down to a value approximately $1 / N$ times the initial one when $\delta=\delta_{M}$, see Fig. 14. In contrast, $\mathrm{g}_{2, c}$ takes its smallest values for $\delta=1$ and $\delta=\delta_{M}$, where it is approximately given by $\mathrm{g}_{2, c}^{\min } \approx 4 E_{C}^{a} / \sqrt{2} E_{C}^{c}$; the largest values at $\delta \sim N / 4$ are bigger by a factor of less than 3 . It turns out (cf. Fig. 15) that for typical experimental parameters even the minimum value $\mathrm{g}_{2, c}^{\min }$ is larger than the strongest collective mode-cavity coupling $g_{1} / 4 E_{C}^{c}$ in Eq. (33) [42]. The contrasting dependence on $\delta$ of the two couplings implies that the position of the coupling capacitors can significantly affect the qubit coherence, as we discuss at the end of the next section.

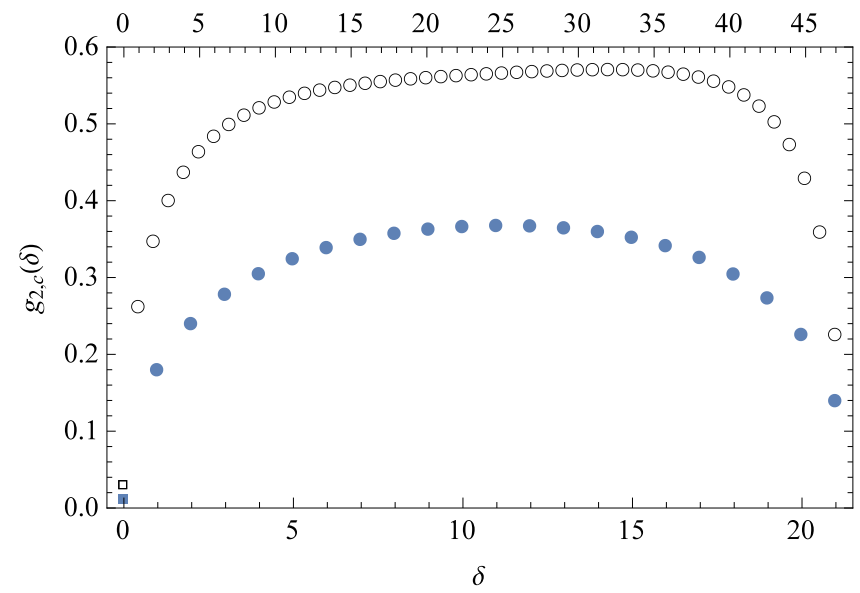

FIG. 15. (Color online) Collective mode-cavity coupling $\mathrm{g}_{2, c}$ [Eq. (83)] vs coupling capacitors position $\delta$. Horizontal bottom (top) scale is used for set 1 , filled circles (2, empty circles). The squares at $\delta=0$ are given by $g_{1} / 4 E_{C}^{c}$ [cf. Eq. (33)], with $g_{1}$ from Eq. (32), and are plotted here for comparison. 


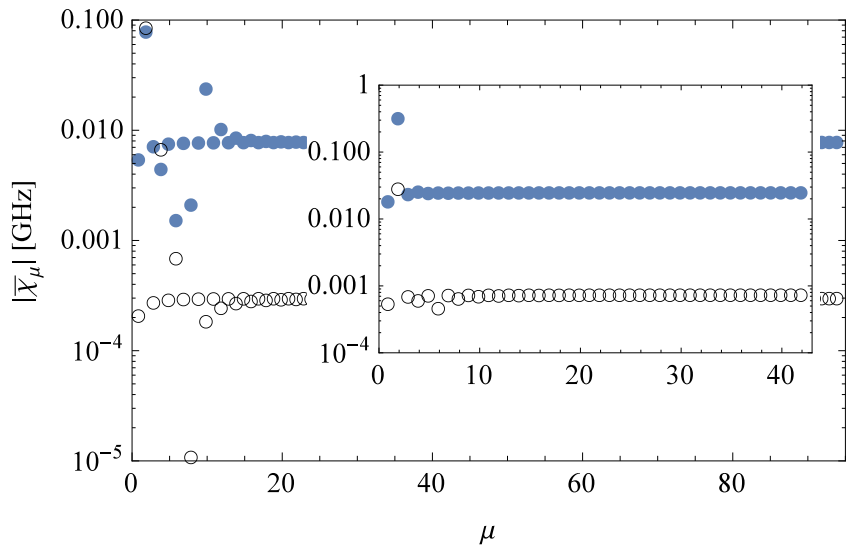

FIG. 16. (Color online) Absolute values of the total dispersive shifts $\left|\bar{\chi}_{\mu}\right|$ for parameter sets 1 (inset) and 2 (main panel). Filled circles are evaluated at zero external flux, empty circles at half-flux quantum. The higher collective modes have very similar values of $\left|\bar{\chi}_{\mu}\right|$, dominated by $\chi_{\mu}^{\delta E_{L}}$ of Eq. (56). The large fluctuations of $\left|\bar{\chi}_{\mu}\right|$ for the low even modes are due to $\chi_{\rho}$ of Eq. (36). In all cases, the lowest even mode $(\mu=2)$ has the largest shift.

\section{ESTIMATES OF QUBIT DEPHASING RATE}

The analysis of the previous sections enables us to estimate the fluxonium dephasing rate due to interactions with the collective modes. We first consider the case of coupling capacitors next to the phase slip-junction, as in Fig. 1; then, we comment on the modifications of our estimates in the case of coupling capacitors farther from the phase-slip junction as in Sec. VII. As explained in Sec. II, for each mode we need to know the corresponding total dispersive shift $\bar{\chi}_{\mu}$; for the circuit of Fig. 1 it is given by

$$
\bar{\chi}_{\mu}= \begin{cases}\chi_{\mu}^{\delta E_{L}}, & \mu \text { odd } \\ \chi_{2 \rho}^{\delta E_{L}}+\chi_{\rho}, & \mu=2 \rho \text { even. }\end{cases}
$$

The contribution due to array junction nonlinearity $\chi_{\mu}^{\delta E_{L}}$ [Eq. (56)] is present for all modes, while that due to ground capacitances $\chi_{\rho}$ [Eq. (36)] only for the even modes. To gauge the relative importance of the two contributions, we note that while $\chi_{\rho}$ quickly decreases in magnitude with increasing index $\rho, \chi_{2 \rho}^{\delta E_{L}}$ slowly increases with $\rho$. Therefore, we can expect that while it may be necessary to keep $\chi_{\rho}$ for the low-index modes, $\chi_{2 \rho}^{\delta E_{L}}$ could be the only relevant contribution for higher-index modes. To identify which modes have low index in this sense, we remind that in the dispersive regime we have $\left|\chi_{\rho}\right| \ll g_{\rho}$, so we can certainly neglect $\chi_{\rho}$ if we find $g_{\rho}<\left|\chi_{2 \rho}^{\delta E_{L}}\right|$. Unfortunately, this latter condition is satisfied at any flux only for high-index modes, so in general we must keep both $\chi_{\rho}$ and $\chi_{2 \rho}^{\delta E_{L}}$ for quantitative estimates (see also Fig. 16). In the remainder of this section we restrict our attention to the flux being zero or half a flux quantum since it is experimentally established [10] that away from these "sweet spots" the fluxonium dephasing rate is determined by flux noise. However, we note that near resonances where the dispersive shifts are enhanced (see Figs. 4 and 5), they could give rise to reproducible suppressions of coherence time $T_{2}$ at specific flux values.
TABLE II. Estimates (in $\mathrm{MHz}$ ) for the dephasing rates $\Gamma_{\phi}^{w}$ [Eq. (88)] and $\Gamma_{\phi}^{\kappa}$ [Eq. (89)]. In contrast to the rate $\Gamma_{\phi}^{\kappa}$, the "worst-case" estimates $\Gamma_{\phi}^{w}$ are not experimentally relevant (see text for details).

\begin{tabular}{lccr}
\hline \hline & $\Gamma_{\phi}^{w}(0)$ & $\Gamma_{\phi}^{w}(0.5)$ & \multicolumn{1}{c}{$\Gamma_{\phi}^{\kappa}$} \\
\hline Set 1 & 13.1 & 0.56 & $1 \times 10^{-6}$ \\
Set 2 & 7.99 & 1.17 & $1.5 \times 10^{-6}$ \\
\hline \hline
\end{tabular}

To determine if the collective modes can at least in principle be a significant source of dephasing, let us consider a worst-case scenario in which all collective modes are equally populated, $\bar{n}_{\mu} \equiv \bar{n}$, and each rate $\Gamma_{\mu}[$ Eq. (2)] attains its maximum value. Then, we have [cf. Eq. (3)]

$$
\Gamma_{\phi}^{w}(f)=\bar{n} \sum_{\mu}\left|\bar{\chi}_{\mu}(f)\right| .
$$

We have calculated $\left|\bar{\chi}_{\mu}\right|$ at zero and half-flux quantum for both parameter sets in Table I (see Fig. 16). Summing over all modes and assuming $\bar{n}=0.01$, we arrive at the results summarized in Table II. We find that in the worst case, the collective modes could limit the dephasing time to about $0.1 \mu$ s at zero flux and $1 \mu \mathrm{s}$ at half-flux quantum. Measured coherence times are longer than these estimates [10,11], indicating that the worst case is not realized in practice. In fact, in Ref. [11] the Purcell-limited lifetime of the qubit at zero flux was measured to be at least $10 \mu \mathrm{s}$, corresponding to a decay rate $\kappa_{q}(0) \sim$ $10^{5} \mathrm{~Hz}$; then, based on the results of Sec. IV C [see in particular Fig. 7 , showing $\kappa_{1} / \kappa_{q}(0) \sim 10^{-3}$ and much smaller ratios for the higher modes] we estimate that the decay rate of the lowest even mode is of order $100 \mathrm{~Hz}$, much smaller than the dispersive shift, and all other modes have even smaller decay rates [43]. Therefore, we conclude that the condition $\kappa_{\mu}=2\left|\chi_{\mu}\right|$ leading to the highest possible dephasing is not met, and that the experimentally relevant regime is that of slow decay of the modes in comparison with the dispersive shift.

In the realistic regime $\kappa_{\mu} \ll\left|\bar{\chi}_{\mu}\right|$, from Eqs. (3) and (4) we find

$$
\Gamma_{\phi}^{\kappa}=\bar{n} \sum_{\mu} \kappa_{\mu} .
$$

Using this formula and Eqs. (41)-(43), we get the estimates in the last column of Table II, corresponding to a dephasing time of order $1 \mathrm{~s}$ (dominated by the relaxation rate of the lowest even collective mode). This time scale is much longer than the coherence times measured in experiments, indicating that most likely the collective modes are not causing any significant dephasing. We caution the reader that the estimates in the last column of Table II rest mainly on the assumption (valid within our model in Fig. 1 for typical parameter values as in Table I) that the collective modes are more weakly coupled to the cavity than the qubit is, as in Fig. 7; if the assumption is not correct, this could result in a dephasing time shorter by several orders of magnitudes, as we discuss next.

We now briefly consider the case in which the coupling capacitors are not placed next to the phase-slip junction (see Fig. 12 in Sec. VII for an example). As the distance $\delta$ between coupling capacitors and phase-slip junction increases, the coupling capacitances must also increase to attain the desired 
qubit-cavity coupling strength (cf. Fig. 14); both moving the capacitors and increasing their capacitance, however, raise the collective mode-cavity coupling, which in turn increases the collective modes decay rates $\kappa_{\mu}$ (cf. Sec. IV C) and hence the qubit dephasing rate (89). We therefore conclude that placing the coupling capacitors beside the phase-slip junction $\delta=0$ is the optimal choice: for a given qubit-cavity coupling strength, this choice minimizes the collective modes decay rates and hence the qubit dephasing.

As a nonoptimal example, let us consider the case in which the coupling capacitors are placed opposite to the phase-slip junction $(\delta \sim N / 2)$. Then, the coupling capacitance should be increased by a factor of order $N$ to compensate for the decrease in qubit-cavity coupling strength. Together with the stronger mode-cavity coupling, $\mathrm{g}_{2, c}^{\min }$ as compared to $g_{1} / 4 E_{C}^{c}$ (Fig. 15), this increase would raise the collective modes decay rate by at least $\sim 10 N^{2}$ (i.e., $\sim 10^{4}$ ). Then, the decay rate of the lowest even mode would be faster than that of the qubit, the dephasing rate $\Gamma_{\phi}^{\kappa}$ of Eq. (89) would also increase by about four orders of magnitudes, and the corresponding dephasing time would be about $0.1 \mathrm{~ms}$. This time is one order of magnitude longer than the coherence time measured in Ref. [11], indicating again that the collective modes are not limiting coherence in current experiments. On the other hand, our estimate is much shorter than the measured $\sim 10 \mathrm{~ms}$ relaxation time at half-flux quantum, so the effect of the collective modes could in principle be observable, if other dephasing mechanisms can be identified and suppressed.

Comparison between the nonoptimal $(\delta \sim N / 2)$ and optimal $(\delta=0)$ cases indicates that if necessary the simplest recipe to decrease the collective mode-induced dephasing rate is to place the coupling capacitors sufficiently close to the phase-slip junction. Alternatively, better filtering could lead to a decrease in the average occupation of the collective modes; in this way the cavity residual occupation was lowered by about one order of magnitude [30], but this gain is much smaller than the four-orders-of-magnitude difference attainable by moving the capacitors.

Finally, let us comment on the reliability of our estimates. As noted towards the end of Sec. VI, a more accurate determination of the array capacitance to ground may show that its value is bigger by a factor of about 3 ; if the value of the ground capacitance was indeed underestimated, the entries in Table II should then be increased by one order of magnitude. This does not affect the qualitative conclusions above for the circuit in Fig. 1 (the worst-case scenario is not realized, and the estimated dephasing rate is many orders of magnitude smaller than the measured one). As for the case of nonoptimal placement of the coupling capacitors, the above considerations are not affected by the value of the ground capacitances since the mode-cavity coupling is dominated by the direct interaction via the coupling capacitors rather than by the interaction mediated by the ground capacitors (in fact, the calculations of Sec. VII are performed in the limit of zero ground capacitances).

\section{SUMMARY}

In this paper, we study the collective modes in the array of Josephson junction forming the superinductance of the fluxonium qubit. We derive an approximate Hamiltonian (29) that includes the interactions between the qubit mode and the collective modes in the presence of ground capacitances. The approximations place some restriction on the number of array junction to which the model applies [see Eq. (28)], but this condition is in practice much weaker than that given by the array "screening length" [Eq. (27)] and it is satisfied in current experiments. A generalization of this Hamiltonian enables us to favorably compare the calculated spectrum of the collective modes to two recent experiments (see Sec. VI and Fig. 11).

In Sec. V, we consider the leading-order nonlinearity of the array junctions, which introduces additional qubitcollective mode interactions. Among these interactions, the term which leads to the strongest dispersive shifts effectively induces fluctuations in the qubit-inductive energy when the collective modes are excited (see Sec. VB). As we discuss in Sec. VIII, the total dispersive shifts (i.e., including also the effect of ground capacitances) are much bigger than the collective mode decay rates, so the latter determine the qubit dephasing rate. We find that the collective modes do not significantly contribute to dephasing, so long as they are more weakly coupled to the cavity than the qubit is; the weak coupling is generically achieved if the qubit-cavity coupling capacitors are placed next to the phase-slip junction. However, we estimate that the collective-mode induced dephasing could become observable if the coupling capacitors are placed opposite to the phase-slip junction. Our findings that the complexity of a circuit does not necessarily affect adversely the qubit coherence is encouraging also for alternative designs of a superinductor [22,23], although a careful analysis of each design is needed to identify its strengths and weaknesses.

\section{ACKNOWLEDGMENTS}

We gratefully acknowledge interesting discussions with D. DiVincenzo, F. Konsçhelle, S. Mehl, I. Pop, G. Rastelli, F. Solgun, and U. Vool. This work was supported in part by the Alexander von Humboldt and Knut och Alice Wallenbergs foundations (G.V.) and by the EU under REA Grant Agreement No. CIG-618258 (G.C.).

\section{APPENDIX A: LAGRANGIAN}

This Appendix provides a derivation of the Lagrangian $\mathcal{L}$ for the collective and qubit modes [Eq. (22)] starting from a more familiar "textbook" formulation in terms of phases and voltages. To this end, we split $\mathcal{L}$ as a sum of kinetic and potential energy parts as usual, $\mathcal{L}=T-U_{S}$, and write the potential energy as

$$
U_{S}=-E_{J}^{a} \sum_{m=1}^{N} \cos \theta_{m}-E_{J}^{b} \cos \left(\sum_{m=1}^{N} \theta_{m}+\varphi_{e}\right) .
$$

Here, $\theta_{m}$ is the (gauge-invariant) phase difference across junction $m$ in the array, and the first term on the right-hand side is the Josephson energy of the array junctions. The last term in the above equation is the phase-slip junction energy, and in writing this term we have taken into account the fluxoid 
quantization condition

$$
\sum_{m=0}^{N} \theta_{m}+\varphi_{e}=2 \pi n
$$

with $n$ integer.

The kinetic energy part $T$ is more easily expressed in terms of the voltages $\dot{\varphi}_{m} / 2 e$ of each island:

$$
\begin{gathered}
T=T_{S}+T_{G}+T_{V}, \\
T_{S}=\sum_{m=1}^{N} \frac{\left(\dot{\varphi}_{m}-\dot{\varphi}_{m-1}\right)^{2}}{16 E_{C}^{a}}+\frac{\left(\dot{\varphi}_{N}-\dot{\varphi}_{0}\right)^{2}}{16 E_{C}^{b}}, \\
T_{G}=\sum_{m=0}^{N} \frac{\dot{\varphi}_{m}^{2}}{16 E_{g}^{m}}, \\
T_{V}=\sum_{m=0}^{N} \frac{\left(\dot{\varphi}_{m}-2 e V_{m}\right)^{2}}{16 E_{c}^{m}} .
\end{gathered}
$$

Here, $T_{S}$ is the charging energy due to the junctions capacitances, $T_{G}$ due to capacitances between each island and ground, and $T_{V}$ due to capacitive coupling to external voltage sources $V_{m}$. In the above equations $E_{g}^{m}=e^{2} / 2 C_{g}^{m}$ and $E_{c}^{m}=e^{2} / 2 C_{c}^{m}$ are charging energies of ground and coupling capacitors for the $m$ th island and they can in general be different for each island. We stress that all equations in this appendix are valid for this generic case, not just for the specific circuit depicted in Fig. 1.

To rewrite $T$ in terms of the phase differences $\theta_{m}$, we use the relationship

$$
\varphi_{m}=\varphi_{0}+\sum_{l=1}^{m} \theta_{l},
$$

valid for $m=1, \ldots, N$, where we have taken $\varphi_{0}$ as a reference phase. Then, it is straightforward to write $T_{S}$ in terms of $\theta_{m}$ variables

$$
T_{S}=\sum_{m=1}^{N} \frac{\dot{\theta}_{m}^{2}}{16 E_{C}^{a}}+\frac{\left(\sum_{m=1}^{N} \dot{\theta}_{m}\right)^{2}}{16 E_{C}^{b}}
$$

The other two terms in $T$ take the form

$$
\begin{gathered}
T_{G}=\frac{\dot{\varphi}_{0}^{2}}{16 E_{g}^{0}}+\sum_{m=1}^{N} \frac{1}{16 E_{g}^{m}}\left(\dot{\varphi}_{0}+\sum_{l=1}^{m} \dot{\theta}_{l}\right)^{2} \\
T_{V}=\frac{\left(\dot{\varphi}_{0}-2 e V_{0}\right)^{2}}{16 E_{c}^{0}}+\sum_{m=1}^{N} \frac{1}{16 E_{c}^{m}}\left(\dot{\varphi}_{0}+\sum_{l=1}^{m} \dot{\theta}_{l}-2 e V_{m}\right)^{2} .
\end{gathered}
$$

We next note that $\mathcal{L}$ is independent of $\varphi_{0}$, so that $\partial \mathcal{L} / \partial \dot{\varphi}_{0}$ is a conserved quantity, the total charge of the circuit [24]. Using this conservation law we can express $\dot{\varphi}_{0}$ in terms of the variables $\theta_{m}$ and thus eliminate it from the Lagrangian. In this way, standard algebraic manipulations lead to

$$
T_{G}=\frac{1}{16} \sum_{m, n=1}^{N} \mathcal{G}_{m n} \dot{\theta}_{m} \dot{\theta}_{n},
$$

$$
\begin{aligned}
\mathcal{G}_{n m} & =E_{t} \sum_{i=0}^{\min \{m, n\}-1} \sum_{j=\max \{m, n\}}^{N}\left(\frac{1}{E_{g}^{i}}+\frac{1}{E_{c}^{i}}\right)\left(\frac{1}{E_{g}^{j}}+\frac{1}{E_{c}^{j}}\right), \\
T_{V} & =\frac{2 e}{8} \sum_{m=1}^{N} \dot{\theta}_{m} \sum_{i=m}^{N}\left[\left(\frac{1}{E_{g}^{i}}+\frac{1}{E_{c}^{i}}\right) \bar{V}-\frac{V_{i}}{E_{c}^{i}}\right], \\
\bar{V} & =E_{t} \sum_{i=0}^{N} \frac{V_{i}}{E_{c}^{i}}
\end{aligned}
$$

where

$$
\frac{1}{E_{t}}=\sum_{i=0}^{N}\left(\frac{1}{E_{g}^{i}}+\frac{1}{E_{c}^{i}}\right) .
$$

These equations correctly reduce to those of [24] in the absence of coupling capacitors.

As a final step, we introduce a new set of variables via the relations

$$
\begin{gathered}
\phi=\sum_{m=1}^{N} \theta_{m}, \\
\xi_{\mu}=\sum_{m=1}^{N} W_{\mu m} \theta_{m}
\end{gathered}
$$

with index $\mu=1, \ldots, N-1$ and inverse $\theta_{m}=\phi / N+$ $\sum_{\mu} W_{\mu m} \xi_{\mu}$. The matrix $W_{\mu m}$ must satisfy the conditions $\sum_{m} W_{\mu m}=0$ and $\sum_{m} W_{\mu m} W_{\nu m}=\delta_{\mu \nu}$. In terms of these new variables we find $T_{S}$ as in Eq. (6) and $U_{S}$ as in Eq. (7). Formulas for $T_{G}$ and $T_{V}$ and arbitrary $W_{\mu m}$ are not instructive, so we do not report them here. For the specific circuit configuration and choice of $W_{\mu m}$ described in Sec. III, the corresponding formulas are given there and follow directly from the equations above. Modifications of those formulas for a different circuit configuration breaking parity symmetry are discussed in Sec. VI. A third circuit with coupling capacitors connected into the array is briefly considered in Sec. VII. Here, we mention a useful identity valid for the choice of $W_{\mu m}$ in Eq. (9):

$$
\sum_{m} m W_{\mu m}=-\frac{1}{\sqrt{2 N}} \frac{c_{\mu}}{s_{\mu}^{2}} o_{\mu}
$$

[for the notation used, see the definitions in Eqs. (18)-(20)].

\section{APPENDIX B: CHOICE OF PARAMETERS}

An often measured property of a flux-tunable qubit such as the fluxonium is its spectrum as a function of flux, $\omega_{10}(f)$. The spectrum can be obtained by numerical diagonalization of the qubit Hamiltonian $H_{\phi}$ [Eq. (30)], where the inductive energy $E_{L}$ should be replaced by $\tilde{E}_{L}$ [Eq. (50)]. For the experiments reported in Refs. $[9,11]$, with $N=43$ and 95 array junctions, respectively, this procedure leads to the parameters reported in Table III. We also give there our rough estimate of the ratio $C_{g}^{b} / C_{g}^{a}$ which is based on the geometry of the devices in the two experiments.

The phase-slip junction Josephson energy $E_{J}^{b}$ in Table I is taken directly from the experimental estimates in Table III. For the coupling capacitor energy $E_{C}^{c}$, we use for set 1 the 
TABLE III. Additional fluxonium parameters estimated as explained in Appendix B. Energies are given in GHz.

\begin{tabular}{lccccc}
\hline \hline & $N$ & $E_{J}^{b}$ & $\tilde{E}_{C}^{\phi}$ & $\tilde{E}_{L}$ & $C_{g}^{b} / C_{g}^{a}$ \\
\hline Set 1 & 43 & 8.93 & 2.39 & 0.52 & 32 \\
Set 2 & 95 & 10.2 & 3.60 & 0.46 & 0.6 \\
\hline \hline
\end{tabular}

value of coupling capacitance given in Ref. [9], while for set 2 we take as an example the value reported in Ref. [18]. The latter experiment was performed with junctions fabricated with the same procedure used to fabricate the fluxonium junctions of [11]; for this reason, we also use the value of ground capacitance in Ref. [18] to estimate $E_{g}^{a}$ for set 2 . The value of $E_{g}^{a}$ for set 1 is smaller than that of set 2 by a factor of 0.4 because, according to the supplement to Ref. [18], the different fabrication processes lead to such a difference in capacitances to ground. Values for $E_{g}^{b}$ then follow from the geometrically estimated ratio $C_{g}^{b} / C_{g}^{a}$. To further constrain the parameters, since the only difference between phase-slip and array junctions is in their area, we assume that their plasma frequency is the same, so that $E_{J}^{a} E_{C}^{a}=E_{J}^{b} E_{C}^{b}$. We then choose the parameters $E_{J}^{a}, E_{C}^{a}$, and $E_{C}^{b}$ as to obtain, using Eqs. (26) and (50), the values of $\tilde{E}_{C}^{\phi}$ and $\tilde{E}_{L}$ reported in Table III.

\section{APPENDIX C: COLLECTIVE MODES: ODD SECTOR}

The collective modes odd under $P T$ symmetry are governed by the Lagrangian

$$
\mathcal{L}_{o}=T_{o}-\frac{1}{2} E_{J}^{a} \sum_{\rho=1}^{N_{o}} \zeta_{\rho}^{2},
$$

where to simplify the notation we introduce $\zeta_{\rho}=\xi_{2 \rho-1}$ and the number of odd modes $N_{o}=\lfloor N / 2\rfloor$ equal to the integer part of $N / 2$. In the kinetic energy term, we separate a purely diagonal term $T_{d}$ independent of $\lambda$ [Eq. (17)], and a term $T_{\lambda}$ proportional to $\lambda$ :

$$
\begin{gathered}
T_{o}=T_{d}+\lambda T_{\lambda}, \\
T_{d}=\frac{1}{16} \sum_{\rho=1}^{N_{o}}\left[\frac{1}{E_{C}^{a}}+\frac{1}{4 E_{g}^{a}} \frac{1}{s_{2 \rho-1}^{2}}\right] \dot{\zeta}_{\rho}^{2}, \\
T_{\lambda}=-\frac{1}{32 E_{g}^{a}} \sum_{\sigma, \rho=1}^{N_{o}} \frac{1}{N(N-1)} \frac{c_{2 \sigma-1} c_{2 \rho-1}}{s_{2 \sigma-1}^{2} s_{2 \rho-1}^{2}} \dot{\zeta}_{\sigma} \dot{\zeta}_{\rho} .
\end{gathered}
$$

If the condition $N \ll N_{s}$ in Eq. (27) is satisfied, the effect of the ground capacitances can be treated perturbatively for all modes and all values of $\lambda$. While the condition is not satisfied in current experiments (cf. Table I), as the order number $\sigma$ of the modes increases, the effect rapidly diminishes [44]; moreover, the off-diagonal part is proportional to $\lambda$, which is typically somewhat smaller than unity. This points to the more general viability of a perturbative approach than suggested by the condition in Eq. (27). Indeed, diagonalization of the kinetic energy matrix $T_{o}$ can be obtained by a rotation that eliminates the off-diagonal terms [note that since the potential energy term in Eq. $(\mathrm{C} 1)$ is quadratic and proportional to the identity matrix, any rotation leaves it unchanged]. Such a rotation can be constructed perturbatively order by order in the parameter $\lambda$, with a procedure analogous to that used to perform a Schrieffer-Wolff transformation to an effective Hamiltonian: we want to obtain an antisymmetric matrix $S=\sum_{n=1} S_{n} \lambda^{n}$ such that the product $e^{-S} T_{o} e^{S}$ is diagonal. Expanding this product up to second order we have

$$
\begin{aligned}
e^{-S} T_{o} e^{S}= & T_{d}+\lambda\left(T_{\lambda}-\left[S_{1}, T_{d}\right]\right) \\
& +\lambda^{2}\left(\frac{1}{2}\left[S_{1},\left[S_{1}, T_{d}\right]\right]-\left[S_{2}, T_{d}\right]-\left[S_{1}, T_{\lambda}\right]\right)+\cdots .
\end{aligned}
$$

To eliminate the off-diagonal terms at order $\lambda$, we take $S_{1}$ to have elements $S_{1, \rho \rho}=0$ and for $\rho \neq \sigma$ :

$$
S_{1, \rho \sigma}=\frac{T_{\lambda, \rho \sigma}}{T_{d, \sigma \sigma}-T_{d, \rho \rho}},
$$

where for example the elements $T_{\lambda, \rho \sigma}$ of matrix $T_{\lambda}$ are defined via $T_{\lambda}=\sum_{\rho, \sigma} T_{\lambda, \rho \sigma} \dot{\zeta}_{\rho} \dot{\zeta}_{\sigma}$. We can similarly choose $S_{2}$ to eliminate the off-diagonal terms at order $\lambda^{2}$. The remaining diagonal elements are then, up to order $\lambda^{2}$,

$$
T_{d, \rho \rho}+\lambda T_{\lambda, \rho \rho}-\frac{\lambda^{2}}{2}\left[S_{1}, T_{\lambda}\right]_{\rho \rho}
$$

The last term is more explicitly written as

$$
\left[S_{1}, T_{\lambda}\right]_{\rho \rho}=2 \sum_{\sigma \neq \rho} \frac{T_{\lambda, \rho \sigma} T_{\lambda, \sigma \rho}}{T_{d, \sigma \sigma}-T_{d, \rho \rho}} .
$$

By substituting the matrix elements into Eq. (C7), we arrive at

$$
T_{o} \simeq \frac{1}{16} \sum_{\rho=1}^{N_{o}} \frac{1}{E_{C, \rho}^{o}} \dot{\tilde{\zeta}}_{\rho}^{2},
$$

where up to second order in $\lambda$ the effective charging energy for the new, rotated modes $\tilde{\zeta}_{\rho}$ is

$$
\begin{aligned}
\frac{1}{E_{C, \rho}^{o}} \simeq & \frac{1}{E_{C}^{a}}+\frac{1}{4 E_{g}^{a}}\left[\frac{1}{s_{2 \rho-1}^{2}}-\lambda \frac{2}{N(N-1)} \frac{c_{2 \rho-1}^{2}}{s_{2 \rho-1}^{4}}\right. \\
& \left.+\left(\lambda \frac{2}{N(N-1)}\right)^{2} \sum_{\sigma \neq \rho} \frac{c_{2 \rho-1}^{2} c_{2 \sigma-1}^{2}}{s_{2 \rho-1}^{2} s_{2 \sigma-1}^{4}-s_{2 \rho-1}^{4} s_{2 \sigma-1}^{2}}\right] .
\end{aligned}
$$

Comparison with numerical diagonalization for a range of experimentally relevant parameters shows that this formula is accurate to a few percent even for the lower-energy modes (and much better accuracy for the high-energy modes), and that the term proportional to $\lambda^{2}$ does not contribute significantly to the effective charging energy, thus validating our perturbative approach. With the Lagrangian in diagonal form, it is straightforward to obtain the energy spectrum of the modes:

$$
\omega_{\rho}^{o}=\sqrt{8 E_{C, \rho}^{o} E_{J}^{a}}
$$

Note that the rotated modes $\tilde{\zeta}_{\rho}$ are related to the original modes $\zeta_{\sigma}$ via $\tilde{\zeta}_{\rho}=\left(e^{S}\right)_{\rho \sigma} \zeta_{\sigma}$. For use in Sec. V A, we define 
the matrix $\Lambda_{\mu \nu}$ that performs the rotation in the odd sector while leaving the even sector unchanged:

$$
\Lambda_{\mu \nu}= \begin{cases}\left(e^{S}\right)_{2 \mu-1,2 \nu-1}, & \mu, \nu \text { odd } \\ \delta_{\mu \nu}, & \mu, v \text { even } \\ 0, & \text { otherwise }\end{cases}
$$

\section{APPENDIX D: EVEN-SECTOR HAMILTONIAN}

The Lagrangian $\mathcal{L}_{e}$ in Eq. (23) has a quadratic kinetic energy part, so that transforming to the Hamiltonian amounts to inverting the $\left(N_{e}+1\right) \times\left(N_{e}+1\right)$ matrix $K=K_{d}+K_{i}$ whose diagonal part $K_{d}$ has entries

$$
\begin{gathered}
K_{d, 00}=\frac{1}{8 \tilde{E}_{C}^{\phi}}, \\
K_{d, \rho \rho}=\frac{1}{8 E_{C, \rho}^{e}}, \quad \rho=1, \ldots, N_{e}
\end{gathered}
$$

and the interaction matrix $K_{i}$ has elements

$$
K_{i, 0 \rho}=K_{i, \rho 0}=-\frac{1}{32 \sqrt{2 N}} \frac{1}{E_{g}^{a}} \frac{c_{2 \rho}}{s_{2 \rho}^{2}}
$$

and all other elements are zero. Indeed, after performing a standard Legendre transformation the kinetic part $H_{K}$ of the Hamiltonian has the form

$$
H_{K}=\frac{1}{2} n K^{-1} n-n K^{-1} v,
$$

where

$$
\begin{gathered}
n=\left(n_{\phi}, n_{1}, \ldots, n_{N_{e}}\right), \\
v=\left(-e V / 4 E_{C}^{c}, 0, \ldots, 0\right)
\end{gathered}
$$

are two $N_{e}+1$-dimensional vectors. We can formally write the inverse matrix as

$$
\begin{aligned}
K^{-1}= & K_{d}^{-1} \frac{1}{1-K_{i} K_{d}^{-1} K_{i} K_{d}^{-1}} \\
& -K_{d}^{-1} K_{i} K_{d}^{-1} \frac{1}{1-K_{i} K_{d}^{-1} K_{i} K_{d}^{-1}}
\end{aligned}
$$

and approximate it as

$$
K^{-1} \approx K_{d}^{-1}-K_{d}^{-1} K_{i} K_{d}^{-1}
$$

provided that entries of matrix $K_{i} K_{d}^{-1} K_{i} K_{d}^{-1}$ are small compared to unity. Given the structure of matrix $K_{i}$, this condition translates into

$$
K_{i, 0 \sigma} K_{i, 0 \rho} K_{d, \rho \rho}^{-1} K_{d, 00}^{-1}=\frac{E_{C, \rho}^{e} \tilde{E}_{C}^{\phi}}{32 N\left(E_{g}^{a}\right)^{2}} \frac{c_{2 \rho}}{s_{2 \rho}^{2}} \frac{c_{2 \sigma}}{s_{2 \sigma}^{2}} \ll 1 .
$$

If the perturbative condition (27) is satisfied, we can approximate $E_{C, \rho}^{e} \approx E_{C}^{a}$ [see Eq. (24)]; using that in order of magnitude $\tilde{E}_{C}^{\phi} \sim E_{C}^{a}$, it then follows that the condition (D9) is also satisfied. More interesting is the opposite regime in which Eq. (27) is violated; then, we can take $E_{C, \rho}^{e} \approx$ $4 E_{g}^{a} s_{2 \rho}^{2}$. Substituting this approximation into Eq. (D9) and expanding the trigonometric functions for small $\rho$ and $\sigma$ (which maximizes the left-hand side), we arrive at Eq. (28). Using Eqs. (D4) and (D8) we arrive at Eqs. (29)-(33).

\section{APPENDIX E: SCHRIEFFER-WOLFF TRANSFORMATION FOR $U_{\phi \xi}^{(2)}$}

In Sec. VB, we have argued that the part proportional to $a_{\mu}^{\dagger} a_{\mu}$ of the potential term $U_{\phi \xi}^{(2)}$ gives rise to fluctuations in the inductive energy and hence to corresponding dispersive shifts; here, we consider the remaining part of $U_{\phi \xi}^{(2)}$ proportional to $a_{\mu}^{\dagger} a_{\mu}^{\dagger}+a_{\mu} a_{\mu}$ :

$$
U_{2}=\sum_{\mu} \sum_{l, j} g_{\mu l j}|l\rangle\langle j|\left(a_{\mu}^{\dagger} a_{\mu}^{\dagger}+a_{\mu} a_{\mu}\right),
$$

where

$$
g_{\mu l j}=\frac{E_{J}^{a} l_{\mu}^{2}}{4}\left\langle l\left|\left(1-\cos \frac{\phi}{N}\right)\right| j\right\rangle \simeq \frac{E_{L} l_{\mu}^{2}}{8 N}\left\langle l\left|\phi^{2}\right| j\right\rangle .
$$

An effective qubit-collective modes Hamiltonian $H_{\text {eff }}$ that includes the effect of this interaction term can be obtained by keeping the next-to-leading order, diagonal part of $e^{-S} H e^{S}$, where

$$
H=H_{\phi}+\sum_{\mu} H_{\mu}+U_{2}
$$

with $H_{\phi}$ of Eq. (30) and $H_{\mu}$ of Eq. (72), and

$$
S=\sum_{\mu} \sum_{l, j} g_{\mu l j}|l\rangle\langle j|\left[\frac{a_{\mu}^{\dagger} a_{\mu}^{\dagger}}{\epsilon_{j}-\epsilon_{l}-2 \omega_{\mu}}+\frac{a_{\mu} a_{\mu}}{\epsilon_{j}-\epsilon_{l}+2 \omega_{\mu}}\right] .
$$

In this way, we find

$$
H_{\text {eff }}=H_{\phi}+\sum_{\mu}\left[H_{\mu}+H_{\phi \mu}\right]
$$

with

$$
\begin{aligned}
H_{\phi \mu}= & -\sum_{l, j}|l\rangle\langle l| g_{\mu l j}^{2}\left[\frac{2\left(\epsilon_{j}-\epsilon_{l}\right)}{\left(\epsilon_{j}-\epsilon_{l}\right)^{2}-\left(2 \omega_{\mu}\right)^{2}} n_{\mu}\left(n_{\mu}-1\right)\right. \\
& \left.+\frac{4}{\epsilon_{j}-\epsilon_{l}+2 \omega_{\mu}}\left(n_{\mu}+\frac{1}{2}\right)\right] .
\end{aligned}
$$

When projected onto the qubit subspace, the last line above gives a small contribution to qubit frequency shift as well as to the dispersive shifts; note that due to our assumption $\omega_{10}<\omega_{\mu}$, the terms in the last line are always finite. To see their smallness, consider the inequalities

$$
\begin{aligned}
& \sum_{j}\left|\frac{g_{\mu 0 j}^{2}}{\epsilon_{j}-\epsilon_{0}+2 \omega_{\mu}} \pm \frac{g_{\mu 1 j}^{2}}{\epsilon_{j}-\epsilon_{1}+2 \omega_{\mu}}\right|<\sum_{j} \frac{g_{\mu 0 j}^{2}+g_{\mu 1 j}^{2}}{\omega_{\mu}} \\
& \quad \simeq\left(\frac{E_{L} \ell_{\mu}^{2}}{8 N}\right)^{2} \frac{1}{\omega_{\mu}}\left[\left|\left\langle 1\left|\phi^{4}\right| 1\right\rangle\right|+\left|\left\langle 0\left|\phi^{4}\right| 0\right\rangle\right|\right] \lesssim \frac{\pi^{4}}{2} \frac{E_{L} \ell_{\mu}^{2}}{N^{3}}
\end{aligned}
$$

In the last step we used that since state $|0\rangle(|1\rangle)$ is mostly localized at potential wells with minima between 0 and $\sim \pm \pi$ $(\sim \pm 2 \pi$ and $\sim \pm \pi)$ we have

$$
\left|\left\langle l\left|\phi^{n}\right| l\right\rangle\right| \lesssim[(l+1) \pi]^{n}, \quad l=0,1 .
$$


The last expression in Eq. (E7) shows that the dispersive shift from the last line of Eq. (E6) is much smaller than $\chi_{\mu}^{\delta E_{L}}$ [see Eqs. (56) and (58)].

The term on the first line in Eq. (E6) can diverge if the resonance condition $\epsilon_{j}-\epsilon_{l}-2 \omega_{\mu}=0$ is met, but this divergence simply signals the breakdown of the perturbative approach when $\epsilon_{j}-\epsilon_{l}-2 \omega_{\mu} \approx g_{\mu l j}$. Therefore, the coefficient of the largest possible contribution from the first line of Eq. (E6) is much smaller in magnitude than $\pi^{2} E_{L} \ell_{\mu}^{2} / 2 N$ (the smallness is due to the matrix element being evaluated between one of the low-energy qubit states $|0\rangle$ or $|1\rangle$ and a state with much higher energy). Hence, we find that even near resonance this term is smaller than $\chi_{\mu}^{\delta E_{L}}$; moreover, one should keep in mind that the latter shifts the qubit frequency if any collective mode has at least one excitation, while the former changes the qubit frequency only if a specific mode (the one near resonance with a qubit transition) is excited at least twice, and the probability for the latter situation to happen is smaller by a factor $\sim \bar{n} / N$ if $\bar{n}$ is the average occupation probability (see also the next Appendix).

\section{APPENDIX F: OCCUPATION PROBABILITIES}

In this Appendix, we comment briefly on the assumed smallness of the occupation probabilities for the collective modes. We assume for simplicity an equilibrium probability for each mode, so that the probability $P_{n_{\rho}}$ of having $n_{\rho}$ excitations in mode $\rho$ is

$$
P_{n_{\rho}}=\frac{1}{1+\bar{n}_{\rho}}\left(\frac{\bar{n}_{\rho}}{1+\bar{n}_{\rho}}\right)^{n_{\rho}},
$$

where $\bar{n}_{\rho}$ is the mode average occupation. For an order-ofmagnitude estimate, we take the latter to be the same for all modes $\bar{n}_{\rho} \equiv \bar{n}$ and small $\bar{n} \ll 1$. Then, the probability $P_{0}$ that none of the $N-1$ collective modes is occupied is simply

$$
P_{0}=\frac{1}{(1+\bar{n})^{N-1}} \simeq 1-(N-1) \bar{n},
$$

while the probabilities $P_{1}$ that one mode has a single excitation, $P_{2}$ that one mode has two excitations, and $P_{1,1}$ that two modes have one excitation are

$$
\begin{gathered}
P_{1}=(N-1) \frac{\bar{n}}{(1+\bar{n})^{N}} \simeq(N-1) \bar{n}, \\
P_{2}=(N-1) \frac{\bar{n}^{2}}{(1+\bar{n})^{N+1}} \simeq(N-1) \bar{n}^{2}, \\
P_{1,1}=\frac{(N-1)(N-2)}{2} \frac{\bar{n}^{2}}{(1+\bar{n})^{N+1}} \simeq \frac{(N-1)^{2}}{2} \bar{n}^{2} .
\end{gathered}
$$

In approximating the above formulas, we assumed $\bar{n} \ll$ $1 / N \ll 1$, and it is evident that under these assumptions we have $P_{2} \ll P_{1,1} \ll P_{1} \ll P_{0}$.

\section{APPENDIX G: DERIVATION OF POTENTIALS $U_{\phi \xi}^{(1)}$ AND $U_{\phi \xi}^{(3)}$ AND CALCULATION OF FREQUENCY CHANGE $\Delta \omega_{10, \mu}$}

The starting point to derive the formulas for $U_{\phi \xi}^{(1)}$ and $U_{\phi \xi}^{(3)}$ [Eqs. (59) and (64), respectively] is the following approximation:

$$
\sum_{m} \sin \left[\sum_{\mu} W_{\mu m} \xi_{\mu}\right] \approx-\frac{1}{6} \sum_{m, \mu, v, \sigma} W_{\mu m} W_{v m} W_{\sigma m} \xi_{\mu} \xi_{\nu} \xi_{\sigma},
$$

where we used the property $\sum_{m} W_{\mu m}=0$ to eliminate the lowest-order contribution. Next, we express the collective mode coordinates in terms of creation/annihilation operators, and after normal ordering we find

$$
\begin{aligned}
\sum_{\mu, v, \sigma} & W_{\mu m} W_{v m} W_{\sigma m} \xi_{\mu} \xi_{\nu} \xi_{\sigma} \\
= & \sum_{\mu, \nu, \sigma} W_{\mu m} W_{v m} W_{\sigma m} \ell_{\mu} \ell_{\nu} \ell_{\sigma} \\
& \times \frac{1}{2 \sqrt{2}}\left[\left(a_{\mu}^{\dagger} a_{\nu}^{\dagger} a_{\sigma}^{\dagger}+3 a_{\mu}^{\dagger} a_{\nu}^{\dagger} a_{\sigma}+\text { H.c. }\right)+3\left(a_{\mu}^{\dagger}+a_{\mu}\right) \delta_{\nu \sigma}\right]
\end{aligned}
$$

where H.c. denotes the Hermitian conjugate. The last term in square brackets gives the linear interaction term $U_{\phi \xi}^{(1)}$ :

$$
U_{\phi \xi}^{(1)}=-\frac{1}{2} E_{J}^{a} \sin \frac{\phi}{N} \sum_{m} \sum_{\mu, v} W_{\mu m} W_{v m}^{2} \frac{\ell_{v}^{2}}{2} \xi_{\mu} .
$$

To proceed further, we note that

$$
\sum_{m} W_{\mu m} W_{v m} W_{\sigma m}=1 / \sqrt{2 N}
$$

if one of three conditions is satisfied:

$$
\begin{aligned}
& \mu+v-\sigma=0, \\
& \mu-v+\sigma=0, \\
& \mu-v-\sigma=0,
\end{aligned}
$$

while

$$
\sum_{m} W_{\mu m} W_{v m} W_{\sigma m}=-1 / \sqrt{2 N}
$$

if

$$
\mu+v+\sigma=2 N
$$

The sum over $m$ vanishes otherwise. For the sum in Eq. (G3) this only leaves two possibilities, $\mu=2 v$ and $2(N-v)$, which imply that $\mu$ must be even. Setting $\mu=2 \rho$ and using the definitions of $\eta_{\rho}$ and $N_{e}$ given after Eq. (23), we can finally write Eq. (G3) in the form given in Eq. (59).

Using again the identities in Eqs. (G4)-(G9), it is straightforward to cast the terms with products of three operators in Eq. (G2) in the form of Eq. (64). Here, we focus on the calculation of $\Delta \omega_{10, \mu}$, the change in frequency due to the interactions in $U_{\phi_{\xi}}^{(3)}$ [Eq. (64)], when a single collective mode is excited. To this end, let us define the corrections $\delta \epsilon_{l, 0}$ and $\delta \epsilon_{l, \bar{\mu}}$ to the qubit energy $\epsilon_{l}$ depending on whether the collective modes are in their ground state or in state $\left|1_{\bar{\mu}}\right\rangle$, respectively. These corrections can be calculated at second 
order in perturbation theory in $U_{\phi \xi}^{(3)}$, for example:

$$
\delta \epsilon_{l, 0}=\sum_{j, f} \frac{\left|\left\langle j, f\left|U_{\phi \xi}^{(3)}\right| l, 0\right\rangle\right|^{2}}{\epsilon_{l}-\epsilon_{j}-E_{f}} .
$$

Here, $|j, f\rangle$ denotes a generic state $|j\rangle$ for the qubit, with energy $\epsilon_{j}$, and some Fock state $|f\rangle$ with energy $E_{f}$ for the collective modes. The similar expression for $\epsilon_{l, \bar{\mu}}$ is

$$
\delta \epsilon_{l, \bar{\mu}}=\sum_{j, f} \frac{\left|\left\langle j, f\left|U_{\phi \bar{\xi}}^{(3)}\right| l, 1_{\bar{\mu}}\right\rangle\right|^{2}}{\epsilon_{l}+\omega_{\bar{\mu}}-\epsilon_{j}-E_{f}} .
$$

In terms of these corrections, the qubit frequency change is

$$
\Delta \omega_{10, \bar{\mu}}=\left(\delta \epsilon_{1, \bar{\mu}}-\delta \epsilon_{0, \bar{\mu}}\right)-\left(\delta \epsilon_{1,0}-\delta \epsilon_{0,0}\right) .
$$

A great simplification in calculating $\Delta \omega_{10, \bar{\mu}}$ is achieved by noticing that in the difference $\delta \epsilon_{l, \bar{\mu}}-\delta \epsilon_{l, 0}$ all contributions originating from terms in $U_{\phi \xi}^{(3)}$ [Eq. (64)], for which none of the indices of the operators in that equation coincide with $\bar{\mu}$ cancel out. In other words, only terms for which at least one index is $\bar{\mu}$ can contribute to the frequency change. Moreover, since the Hermitian conjugate terms in Eq. (64) contain two or more annihilation operators, they also do not contribute to $\Delta \omega_{10, \bar{\mu}}$. To concretely calculate the matrix elements entering
Eq. (G11), we need to consider the action of the terms in square brackets in Eq. (64) onto the singly excited state $\left|1_{\bar{\mu}}\right\rangle$; for example, the first one gives

$$
\begin{aligned}
& \sum_{\mu, \nu} a_{\mu+\nu}^{\dagger} a_{\mu}^{\dagger} a_{\nu}^{\dagger}\left|1_{\bar{\mu}}\right\rangle \\
& =\sum_{\mu, \nu}^{\prime}\left|1_{\bar{\mu}} 1_{\mu} 1_{\nu} 1_{\mu+\nu}\right\rangle+2 \sum_{\nu}^{\prime} \sqrt{2}\left|2_{\bar{\mu}} 1_{\bar{\mu}+\nu} 1_{\nu}\right\rangle \\
& \quad+\sum_{\nu}^{\prime} \sqrt{2}\left|2_{\bar{\mu}} 1_{\bar{\mu}-\nu} 1_{\nu}\right\rangle+\sqrt{6}\left|3_{\bar{\mu}} 1_{2 \bar{\mu}}\right\rangle+(\sqrt{2})^{2}\left|2_{\bar{\mu}} 2_{\bar{\mu} / 2}\right\rangle,
\end{aligned}
$$

where the prime at the summation symbols implies that all the collective mode indices in the states being summed must be different. The first term on the right-hand side is the one in which no index coincides with $\bar{\mu}$ and, as discussed above, this term does not contribute to the difference $\delta \epsilon_{l, \bar{u}}-\delta \epsilon_{l, 0}$. The last two terms arise from particular combination of the indices ( $\mu=v=\bar{\mu}$ and $\mu=v=\bar{\mu} / 2$, respectively); the last one is present only if $\bar{\mu}$ is even. Similar terms in which two indices are equal also arise from the other operators in square brackets in Eq. (64) [45]. However, we discard these terms in comparison with the terms with sum over index $v$ [see the second line in Eq. (G13)]: because of the sums, the discarded terms are smaller by a factor of order $1 / N$. Taking these considerations into account, standard calculation of the matrix elements for the collective modes gives

$$
\begin{aligned}
\delta \epsilon_{l, \bar{\mu}}-\delta \epsilon_{l, 0} \approx & \frac{\left(E_{J}^{a}\right)^{2}}{16 N} \sum_{j}\left|\left\langle j\left|\sin \frac{\phi}{N}\right| l\right\rangle\right|^{2} \ell_{\bar{\mu}}^{2}\left[\sum_{\nu=1}^{N-\bar{\mu}-1} \ell_{\nu}^{2} \ell_{\bar{\mu}+v}^{2}\left(\frac{1}{\epsilon_{l}-\epsilon_{j}-\omega_{\bar{\mu}}-\omega_{\nu}-\omega_{\bar{\mu}+\nu}}+\frac{1}{\epsilon_{l}-\epsilon_{j}+\omega_{\bar{\mu}}-\omega_{\nu}-\omega_{\bar{\mu}+\nu}}\right)\right. \\
& +\frac{1}{4} \sum_{\nu=1}^{\bar{\mu}-1} \ell_{\nu}^{2} \ell_{\bar{\mu}-v}^{2}\left(\frac{1}{\epsilon_{l}-\epsilon_{j}-\omega_{\bar{\mu}}-\omega_{\nu}-\omega_{\bar{\mu}-\nu}}+\frac{1}{\epsilon_{l}-\epsilon_{j}+\omega_{\bar{\mu}}-\omega_{\nu}-\omega_{\bar{\mu}-\nu}}\right) \\
& \left.+\frac{1}{4} \sum_{\nu=N-\bar{\mu}+1}^{N-1} \ell_{\nu}^{2} \ell_{2 N-\bar{\mu}-v}^{2}\left(\frac{1}{\epsilon_{l}-\epsilon_{j}-\omega_{\bar{\mu}}-\omega_{\nu}-\omega_{2 N-\bar{\mu}-\nu}}+\frac{1}{\epsilon_{l}-\epsilon_{j}+\omega_{\bar{\mu}}-\omega_{\nu}-\omega_{2 N-\bar{\mu}-\nu}}\right)\right],
\end{aligned}
$$

where the approximate equality indicates that we are neglecting $1 / N$ corrections originating from terms like the last two in Eq. (G13).

To estimate a bound on the frequency change $\Delta \omega_{10, \bar{\mu}}$, we note that for $l=0,1$ so long as $\omega_{10}<2 \omega_{1}-\omega_{N-1}$ there are no divergent contributions in Eq. (G14) and the second terms in round brackets are larger than the first ones. For an order-of-magnitude estimate, we further neglect the dependence of the oscillator lengths $\ell_{\mu}$ and collective mode frequencies $\omega_{\mu}$ on the array ground capacitance and substitute in Eq. (G14) the values $\ell_{0}$ and $\omega_{p}^{a}=\sqrt{8 E_{C}^{a} E_{J}^{a}}$, respectively. In this way, we find (for $l=0,1$ )

$$
\left|\delta \epsilon_{l, \bar{\mu}}-\delta \epsilon_{l, 0}\right| \lesssim \frac{E_{L}}{16} \frac{E_{J}^{a}}{N^{2}} \ell_{0}^{6} \sum_{j}\left|\left\langle j\left|N \sin \frac{\phi}{N}\right| l\right\rangle\right|^{2}\left[\left(\sum_{\nu=1}^{N-\bar{\mu}-1}+\frac{1}{4} \sum_{\nu=1}^{\bar{\mu}-1}+\frac{1}{4} \sum_{\nu=N-\bar{\mu}+1}^{N-1}\right) \frac{2}{\omega_{p}^{a}+\left(\epsilon_{j}-\epsilon_{l}\right)}\right] .
$$

With the performed approximations, there is no dependence on collective mode indices, so that the sums in round brackets can be bounded by $N$. An upper bound for the term in square brackets is then $2 N / \omega_{p}^{a}$ [46]. Finally, we approximate the qubit matrix element as follows:

$$
\sum_{j}\left|\left\langle j\left|N \sin \frac{\phi}{N}\right| l\right\rangle\right|^{2} \approx\left|\left\langle l\left|\phi^{2}\right| l\right\rangle\right|
$$


Rough upper bounds for the latter matrix elements are $(2 \pi)^{2}$ for $l=1$ and $\pi^{2}$ for $l=0$ [see Eq. (E8)]. We thus find that the bound is tighter for the $l=0$ correction compared to the $l=1$ one, and hence

$$
\left|\Delta \omega_{10, \bar{\mu}}\right| \leqslant 2\left|\delta \epsilon_{l, \bar{\mu}}-\delta \epsilon_{l, 0}\right| \lesssim \frac{\pi^{2}}{2} \frac{E_{L}}{N} \frac{E_{J}^{a}}{\omega_{p}^{a}} \ell_{0}^{6} .
$$

We now compare the frequency change $\Delta \omega_{10, \bar{\mu}}$ with that due to the dispersive shift $\chi_{\mu}^{\delta E_{L}}$ originating from the quadratic interaction $U_{\phi \xi}^{(2)}$ [see Eq. (56)]; the latter frequency change is given, in order of magnitude, by the upper bound in Eq. (58), so that an approximate bound on the ratio between the two quantities is

$$
\frac{\left|\Delta \omega_{10, \bar{\mu}}\right|}{2 \chi_{\mu}^{\delta E_{L}}} \lesssim\left(\pi \ell_{0}\right)^{2} \frac{E_{L}}{\omega_{10}},
$$

where we used $E_{J}^{a} / \omega_{p}^{a}=\ell_{0}^{-2}$. For typical experimental parameters, the right-hand side is at most of order unity for any value of flux. Given that our approximations place a loose bound on $\Delta \omega_{10, \bar{\mu}}$, we conclude that the latter can generally be neglected in comparison with $\chi_{\mu}^{\delta E_{L}}$.

\section{APPENDIX H: DERIVATION OF EQ. (78)}

For the circuit considered in Sec. VII (symmetrically placed coupling capacitors and no ground capacitances), the matrix $G$ entering Eq. (12) takes the form

$$
\begin{gathered}
G_{00}=\frac{1}{2 E_{C}^{c}}\left(1-\frac{2 \delta}{N}\right)^{2}, \\
G_{0 \mu}=-\frac{1}{E_{C}^{c}}\left(1-\frac{2 \delta}{N}\right) \frac{1}{\sqrt{2 N}} \frac{s_{2 \mu \delta}}{s_{\mu}} o_{\mu+1}, \\
G_{\mu \nu}=\frac{1}{E_{C}^{c}} \frac{1}{N} \frac{s_{2 \mu \delta}}{s_{\mu}} \frac{s_{2 \nu \delta}}{s_{\nu}} o_{\mu+1} o_{v+1} .
\end{gathered}
$$

Note that both matrix $G$ and $\mathcal{L}_{V}$ in Eq. (77) vanish for $\delta=N / 2$; this choice (possible only for even $N$ ) connects both capacitors to the same island, and the vanishing is due to the arbitrariness in choosing a reference for the electric potential. For generic $0<\delta<N / 2$ we see that, beside a renormalization of the qubit charging energy [Eq. (H1)], the coupling capacitors couple the qubit and even modes [Eq. (H2)], similarly to the effect of ground capacitances $C_{g}^{a}$ in the array, while at variance with the effect of the ground capacitances, the coupling capacitors lead to mode-mode interaction in the even sector [Eq. (H3)], rather than in the odd one. (Of course, parity symmetry still guarantees the qubit-odd mode decoupling.) The situation, however, is only apparently complicated since a change of variables shows that the problem reduces to a single collective mode coupled to the qubit and the cavity, while all other modes remain degenerate and decoupled. Indeed, let us introduce the new even-sector variables:

$$
\begin{gathered}
\tilde{\eta}_{1}=\sum_{\rho=1}^{N_{e}} \frac{v_{\rho}}{|v|} \eta_{\rho}, \\
\tilde{\eta}_{\rho}=\frac{|v|_{\rho-1}}{|v|_{\rho}} \eta_{\rho}-\sum_{\sigma=1}^{\rho-1} \frac{v_{\rho} v_{\sigma}}{|v|_{\rho}|v|_{\rho-1}} \eta_{\sigma} .
\end{gathered}
$$

This transformation is a rotation for any vector $v$ with the definition

$$
|v|_{\rho}^{2}=\sum_{\sigma=1}^{\rho} v_{\sigma}^{2}
$$

for the norms, and $|v| \equiv|v|_{N_{e}}$. In the present case, we take the components $v_{\rho}$ to be

$$
v_{\rho}=\frac{1}{\sqrt{N}} \frac{s_{4 \rho \delta}}{s_{2 \rho}}
$$

and find that the kinetic energy term $T_{G}$ simplifies to

$$
T_{G}=\frac{1}{16}\left[G_{00} \dot{\phi}^{2}+\frac{|v|^{2}}{E_{C}^{c}} \dot{\tilde{\eta}}_{1}^{2}-2 \frac{1-2 \delta / N}{\sqrt{2} E_{C}^{c}}|v| \dot{\tilde{\eta}}_{1} \dot{\phi}\right] .
$$

Similarly, $\mathcal{L}_{V}$ of Eq. (77) becomes

$$
\mathcal{L}_{V}=-\frac{1}{4 E_{C}^{c}}\left(1-\frac{2 \delta}{N}\right) \dot{\phi} e V+\frac{1}{2 \sqrt{2} E_{C}^{c}}|v| \dot{\tilde{\eta}}_{1} e V .
$$

The norm of vector $v$ can be calculated explicitly [47]:

$$
|v|^{2}=\delta\left(1-\frac{2 \delta}{N}\right)
$$

Neglecting the array nonlinearities, the total Lagrangian is the sum of $\mathcal{L}_{U}$ in Eq. (11) with $T_{G}$ and $\mathcal{L}_{V}$ of Eqs. (H8) and (H9). Then, performing the Legendre transform and defining

$$
\xi_{\mu}= \begin{cases}\tilde{\eta}_{\mu / 2}, & \mu \text { even } \\ \zeta_{(\mu+1) / 2}, & \mu \text { odd }\end{cases}
$$

for $\mu=1, \ldots, N-1$, we arrive at the Hamiltonian in Eq. (78).
[1] Y. Nakamura, Yu. A. Pashkin, and J. S. Tsai, Nature (London) 398, 786 (1999).

[2] M. H. Devoret and R. J. Schoelkopf, Science 339, 1169 (2013).

[3] H. Paik, D. I. Schuster, L. S. Bishop, G. Kirchmair, G. Catelani, A. P. Sears, B. R. Johnson, M. J. Reagor, L. Frunzio, L. I. Glazman, S. M. Girvin, M. H. Devoret, and R. J. Schoelkopf, Phys. Rev. Lett. 107, 240501 (2011).

[4] R. Barends, J. Kelly, A. Megrant, D. Sank, E. Jeffrey, Y. Chen, Y. Yin, B. Chiaro, J. Mutus, C. Neill, P. O’Malley, P. Roushan, J. Wenner, T. C. White, A. N. Cleland, and J. M. Martinis, Phys. Rev. Lett. 111, 080502 (2013).
[5] M. D. Reed, L. DiCarlo, S. E. Nigg, L. Sun, L. Frunzio, S. M. Girvin, and R. J. Schoelkopf, Nature (London) 482, 382 (2012).

[6] J. Kelly, R. Barends, A. G. Fowler, A. Megrant, E. Jeffrey, T. C. White, D. Sank, J. Y. Mutus, B. Campbell, Yu Chen, Z. Chen, B. Chiaro, A. Dunsworth, I.-C. Hoi, C. Neill, P. J. J. OMalley, C. Quintana, P. Roushan, A. Vainsencher, J. Wenner, A. N. Cleland, and J. M. Martinis, Nature (London) 519, 66 (2015).

[7] A. D. Corcoles, E. Magesan, S. J. Srinivasan, A. W. Cross, M. Steffen, J. M. Gambetta, and J. M. Chow, Nat. Commun. 6, 6979 (2015). 
[8] D. Ristè, S. Poletto, M.-Z. Huang, A. Bruno, V. Vesterinen, O.-P. Saira, and L. DiCarlo, Nat. Commun. 6, 6983 (2015).

[9] V. E. Manucharyan, J. Koch, L. I. Glazman, and M. H. Devoret, Science 326, 113 (2009).

[10] V. E. Manucharyan, N. A. Masluk, A. Kamal, J. Koch, L. I. Glazman, and M. H. Devoret, Phys. Rev. B 85, 024521 (2012).

[11] I. M. Pop, K. Geerlings, G. Catelani, R. J. Schoelkopf, L. I. Glazman, and M. H. Devoret, Nature (London) 508, 369 (2014).

[12] R. M. Bradley and S. Doniach, Phys. Rev. B 30, 1138 (1984).

[13] E. Chow, P. Delsing, and D. B. Haviland, Phys. Rev. Lett. 81, 204 (1998).

[14] G. Rastelli, I. M. Pop, and F. W. J. Hekking, Phys. Rev. B 87, 174513 (2013).

[15] R. Susstrunk, I. Garate, and L. I. Glazman, Phys. Rev. B 88, 060506(R) (2013).

[16] G. Rastelli, M. Vanevic, and W. Belzig, New J. Phys. 17, 053026 (2015).

[17] D. M. Basko and F. W. J. Hekking, Phys. Rev. B 88, 094507 (2013).

[18] N. A. Masluk, I. M. Pop, A. Kamal, Z. K. Minev, and M. H. Devoret, Phys. Rev. Lett. 109, 137002 (2012).

[19] B. Douçot and L. B. Ioffe, Rep. Prog. Phys. 75, 072001 (2012).

[20] P. Brooks, A. Kitaev, and J. Preskill, Phys. Rev. A 87, 052306 (2013).

[21] J. M. Dempster, B. Fu, D. G. Ferguson, D. I. Schuster, and J. Koch, Phys. Rev. B 90, 094518 (2014).

[22] M. T. Bell, I. A. Sadovskyy, L. B. Ioffe, A. Yu. Kitaev, and M. E. Gershenson, Phys. Rev. Lett. 109, 137003 (2012).

[23] M. T. Bell, W. Zhang, L. B. Ioffe, and M. E. Gershenson, arXiv:1504.05602.

[24] D. G. Ferguson, A. A. Houck, and J. Koch, Phys. Rev. X 3, 011003 (2013).

[25] G. Catelani, R. J. Schoelkopf, M. H. Devoret, and L. I. Glazman, Phys. Rev. B 84, 064517 (2011).

[26] G. Catelani, S. E. Nigg, S. M. Girvin, R. J. Schoelkopf, and L. I. Glazman, Phys. Rev. B 86, 184514 (2012).

[27] S. Spilla, F. Hassler, A. Napoli, and J. Splettstoesser, New J. Phys. 17, 065012 (2015).

[28] D. I. Schuster, A. Wallraff, A. Blais, L. Frunzio, R.-S. Huang, J. Majer, S. M. Girvin, and R. J. Schoelkopf, Phys. Rev. Lett. 94, 123602 (2005).

[29] J. Gambetta, A. Blais, D. I. Schuster, A. Wallraff, L. Frunzio, J. Majer, M. H. Devoret, S. M. Girvin, and R. J. Schoelkopf, Phys. Rev. A 74, 042318 (2006).

[30] A. P. Sears, A. Petrenko, G. Catelani, L. Sun, H. Paik, G. Kirchmair, L. Frunzio, L. I. Glazman, S. M. Girvin, and R. J. Schoelkopf, Phys. Rev. B 86, 180504(R) (2012).

[31] C. Rigetti, J. M. Gambetta, S. Poletto, B. L. T. Plourde, J. M. Chow, A. D. Córcoles, J. A. Smolin, S. T. Merkel, J. R. Rozen, G. A. Keefe, M. B. Rothwell, M. B. Ketchen, and M. Steffen, Phys. Rev. B 86, 100506(R) (2012).

[32] We note that in fact Eq. (2) is more generally valid for $\bar{n}_{\mu} \ll$ $\max \left\{1,2 \bar{\chi}_{\mu} / \kappa_{\mu}\right\}$.
[33] As explained before Eq. (10), the approximation consists in neglecting the array junction nonlinearities. We will consider their effects and the resulting interactions between even and odd sectors in Sec. V. Here, we note that the nonlinearities preserve $P T$ symmetry and therefore cannot introduce even/odd couplings at the quadratic level.

[34] G. Zhu, D. G. Ferguson, V. E. Manucharyan, and J. Koch, Phys. Rev. B 87, 024510 (2013).

[35] J. Koch, T. M. Yu, J. Gambetta, A. A. Houck, D. I. Schuster, J. Majer, A. Blais, M. H. Devoret, S. M. Girvin, and R. J. Schoelkopf, Phys. Rev. A 76, 042319 (2007).

[36] We consider low occupation of the collective modes, in which case this condition agrees with the more precise one discussed in Ref. [34].

[37] Identities with the structure of Eq. (63) are generally valid for Hamiltonians with quadratic kinetic energy terms, as can be shown by calculating $|\langle m|[H, \phi]| l\rangle|^{2}$ either by evaluating the commutator first, $[H, \phi] \propto p_{\phi}$, or by applying the Hamiltonian to the eigenstates.

[38] The exceptions are narrow regions in flux around those points where the shift $\chi_{\rho}$ has zeros.

[39] Asymmetry in the ground capacitances $C_{g}^{b}$ next to the phase-slip junction can be accounted for by the replacements $\delta c \rightarrow \delta c+$ $\delta c_{b}$ in Eqs. (67) and (68), with $\delta c_{b}=\left(C_{g}^{0}-C_{g}^{N}\right) / C_{t}$, as well as $(\delta c)^{2} \rightarrow \delta c\left(\delta c+\delta c_{b}\right)$ in the second term of Eq. (69).

[40] T. Weissl, B. Küng, E. Dumur, A. K. Feofanov, I. Matei, C. Naud, O. Buisson, F. W. J. Hekking, and W. Guichard, Phys. Rev. B 92, 104508 (2015).

[41] U. Vool, I. M. Pop, K. Sliwa, B. Abdo, C. Wang, T. Brecht, Y. Y. Gao, S. Shankar, M. Hatridge, G. Catelani, M. Mirrahimi, L. Frunzio, R. J. Schoelkopf, L. I. Glazman, and M. H. Devoret, Phys. Rev. Lett. 113, 247001 (2014).

[42] This relationship will eventually fail as $N$ increases to saturate the bound in Eq. (28).

[43] The odd modes being decoupled, their decay rate is formally zero. As we show in Sec. VI, unavoidable deviation from perfect parity symmetry introduces couplings between the odd modes and the cavity, but these are usually negligible.

[44] Modes with index $\sigma \gg \sigma^{*}=(N / 2) \sqrt{E_{C}^{a} / 4 E_{g}^{a}}$ can always be treated perturbatively; for parameters in Table I we find $\sigma^{*} \approx 1$, so most modes, except the few lowest ones, are in fact in the perturbative regime.

[45] For the last two terms in square brackets in Eq. (64), if $N$ is multiple of 3 then for $\bar{\mu}=2 N / 3$ all indices can be equal.

[46] Strictly speaking, for $l=1$ the upper bound for the term with $j=0$ in the sum over $j$ is $2 N /\left(\omega_{p}^{a}-\omega_{10}\right)$. However, we note that even at zero external flux, the qubit frequency is in practice sufficiently smaller than the collective mode frequency that our neglecting $\omega_{10}$ is justified for an order-of-magnitude estimate. Moreover, when comparing to $\chi_{\mu}^{\delta E_{L}}$ in Eq. (G18), the possible underestimation of $\Delta \omega_{10, \bar{\mu}}$ due to this approximation is compensated for by the prefactor $E_{L} / \omega_{10}$, which is minimum at zero external flux.

[47] A. Gervois and M. L. Mehta, J. Math. Phys. 36, 5098 (1995). 